\title{
MERGING GALAXY CLUSTER A2255 IN MID-INFRARED
}

\author{
Hyunjin Shim ${ }^{1,2,3}$, Myungshin Im²,3, Hyung Mok Lee ${ }^{2}$, Myung Gyoon LeE $^{2}$, Seong Jin Kim ${ }^{2}$, Ho Seong Hwang ${ }^{4}$, \\ Narae Hwang ${ }^{5}$, Jongwan Ko ${ }^{2,3}$, Jong Chul Lee ${ }^{2}$, Sungsoon Lim² ${ }^{2}$, Hideo Matsuhara ${ }^{6}$, Hyunjong SeO 2 , \\ TAKehiko Wada ${ }^{6}$, AND Tomotsugu Goto ${ }^{7}$ \\ ${ }^{1}$ Spitzer Science Center, California Institute of Technology, MS 220-6, Pasadena, CA 91125, USA; hjshim @ipac.caltech.edu \\ 2 Astronomy Program, Department of Physics and Astronomy, FPRD, Seoul National University, Seoul, Republic of Korea \\ ${ }^{3}$ Center for the Exploration of the Origin of the Universe (CEOU), Seoul National University, Seoul, Republic of Korea \\ ${ }^{4}$ CEA Saclay/Service d'Astrophysique, F-91191 Gif-sur-Yvette, France \\ ${ }^{5}$ National Astronomical Observatory of Japan, Mitaka, Tokyo 181-8588, Japan \\ ${ }^{6}$ Institute of Space and Astronautical Science, Japan Aerospace Exploration Agency, Kanagawa 229-8510, Japan \\ ${ }^{7}$ Institute for Astronomy, University of Hawaii, 2680 Woodlawn Drive, Honolulu, HT 96822, USA \\ Received 2009 August 14; accepted 2010 November 4; published 2010 December 23
}

\begin{abstract}
We present the mid-infrared (MIR) observation of a nearby galaxy cluster, A2255, by the $A K A R I$ space telescope. Using AKARI's continuous wavelength coverage between 3 and $24 \mu \mathrm{m}$ and the wide field of view, we investigate the properties of cluster member galaxies to see how the infall of the galaxies, the cluster substructures, and the cluster-cluster merger influence their evolution. We show that the excess of MIR $(\sim 11 \mu \mathrm{m})$ flux is a good indicator for discriminating galaxies at different evolutionary stages and for dividing galaxies into three classes accordingly: strong MIR-excess $(N 3-S 11>0.2)$ galaxies that include both unobscured and obscured star-forming galaxies; weak MIR-excess $(-2.0<N 3-S 11<-1.2)$ galaxies that are quiescent, old ( $>5$ Gyr) galaxies where the MIR emission arises mainly from the circumstellar dust around AGB stars; and intermediate MIR-excess $(-1.2<N 3-S 11<0.2)$ galaxies in between the two classes that are less than a few Gyr old past the prime star formation activity. With the MIR-excess diagnostics, we investigate how local and cluster-scale environments affect the individual galaxies. We derive the total star formation rate (SFR) and the specific SFR of A2255 using the strong MIR-excess galaxies. The dust-free, total SFR of A2255 is $\sim 130 M_{\odot} \mathrm{yr}^{-1}$, which is consistent with the SFRs of other clusters of galaxies at similar redshifts and with similar masses. We find no strong evidence that supports enhanced star formation either inside the cluster or in the substructure region, suggesting that the infall or the cluster merging activities tend to suppress star formation. The intermediate MIR-excess galaxies, representing galaxies in transition from star-forming galaxies to quiescent galaxies, are located preferentially at the medium density region or cluster substructures with higher surface density of galaxies. Our findings suggest that galaxies are being transformed from star-forming galaxies into red, quiescent galaxies from the infall region through near the core which can be explained well by ram-pressure stripping as previous simulation results suggest. We conclude that the cluster merging and the group/galaxy infall suppress star formation and transform galaxies from star-forming galaxies into quiescent galaxies, most likely due to ram-pressure stripping.
\end{abstract}

Key words: galaxies: clusters: individual (A2255) - galaxies: photometry - infrared: galaxies

Online-only material: color figures, machine-readable table

\section{INTRODUCTION}

The formation and the evolution of galaxies are thought to be strongly dependent on their environments. As an extreme example of a high-density environment, galaxy clusters may affect star formation activities of their member galaxies through various processes (Boselli \& Gavazzi 2006; Park \& Hwang 2009), including ram-pressure stripping (Gunn \& Gott 1972; Abadi et al. 1999), cluster tidal forces (Fujita 1998), violent galaxy encounters (Lavery \& Henry 1994), or rapid galaxy encounters such as galaxy harassment (Moore et al. 1996). These mechanisms are considered to be the reasons why galaxy clusters show less star formation than field both at low and high redshifts (e.g., Dressler \& Gunn 1983; Poggianti et al. 1999, 2009).

To date, a number of galaxy clusters are known to be in a merging process through their asymmetric X-ray emission and substructures in member distribution (e.g., Markevitch et al. 1998; Donnelly et al. 2001). The effect of cluster-scale merging on the member galaxies is still being debated (e.g., Hwang \& Lee 2009): the cluster-cluster merging can trigger star formation in member galaxies by driving the external potential (e.g., Bekki 1999), or quench star formation by depriving gas via ram pressure of the intracluster medium (e.g., Fujita et al. 1999). Both hypotheses are supported by observations, but so far it is unclear which case is more dominant.

Abell 2255 (hereafter A2255) is a rich galaxy cluster at low redshift $(z=0.0806$; Struble \& Rood 1999) that consists of a few hundred member galaxies. Due to its richness (richness class 2; Abell 1958) and the advantage of extensive membership studies (cluster membership is determined partly by using the spectroscopic redshifts from the Sloan Digital Sky Survey Data Release 2 (SDSS DR2), Abazajian et al. 2004; and partly by using the photometric redshifts by Yuan et al. 2003), the cluster is ideal for investigation of stellar populations and evolution of its member galaxies. Previous studies based on various wavelengths$\mathrm{X}$-ray, optical, and radio-have suggested that A2255 is not a relaxed cluster, but in a process of cluster-cluster merging. The X-ray contour map is elongated in the east-west direction which implies that there was a merger in this direction (Davis \& White 1998; Feretti et al. 1997). Since there are no distinct two peaks in the X-ray temperature distribution, it seems that 
A2255 is already in the late stage of the cluster-cluster merger. Optical member identification-using spectroscopic and photometric redshifts-shows that A2255 has several significant substructures with different velocity components (Yuan et al. 2003). These substructures are interpreted as groups of galaxies infalling into the main cluster. Radio observations revealed an excess of radio galaxies in A2255 (Miller \& Owen 2003), some of them active galactic nucleus (AGN) dominated. Davis et al. (2003) found X-ray point sources in A2255 that are thought to be AGNs, and they also found that the total number of AGNs in A2255 is significantly larger than that of a typical galaxy cluster. Finally, a possible alignment of star-forming galaxies that prefer a specific direction (south-north; Miller \& Owen 2003) can also be interpreted as the result of the cluster-cluster merger. All these observations support the idea that A2255 is a good example of an "unrelaxed" galaxy cluster.

Yuan et al. (2005) discussed the effects of cluster-cluster merging on the evolution of member galaxies using SDSS spectroscopy. From the morphological analysis, they suggested that the cluster-cluster merger has different effects on the star formation activity of galaxies with different morphologies: star formation activity is suppressed in E/SO galaxies and enhanced in spiral and irregular galaxies.

However, this previous study on the star formation of member galaxies is limited to the optically bright galaxies with spectroscopic information. It is not clear whether these optically bright galaxies account for the majority of star formation taking place in A2255. First, there could be member galaxies that contribute significantly to the total ongoing star formation despite their faint magnitudes in the optical. Second, there could be heavily obscured star formation that is not accounted for with the extinction correction estimated from the optical emission line ratios, as witnessed by recent infrared (IR) studies of the obscured star formation in the core of several clusters of galaxies (e.g., Bai et al. 2007; Marcillac et al. 2007). The mid-infrared (MIR) emission is an efficient probe of these "hidden" star formations, since it is proportional to the total IR luminosity (e.g., Chary \& Elbaz 2001). The MIR emission between 3 and $10 \mu \mathrm{m}$ also plays an important role in detecting the existence of dust inside galaxies (Bressan et al. 2007), which provides hints to the evolution of galaxies.

In this paper, we present 3-24 $\mu \mathrm{m}$ observation of $\sim 1200$ $\operatorname{arcmin}^{2}$ over A2255 field using the IR telescope AKARI. By combining $A K A R I$ MIR data with the optical/X-ray/radio data and Spitzer $24 / 70 \mu \mathrm{m}$ data, we study the properties of cluster member galaxies in order to investigate the effect of the cluster-cluster merger on their evolution. In particular, we show that a significant fraction of cluster member galaxies have "excess" in MIR with respect to the photospheric emission, i.e., emission from stellar photosphere. The MIR-excess proves the presence of dust emission in these galaxies, which is an important sign of galaxy evolution. Thus, we focus on these "MIR-excess" galaxies and investigate their properties in relation to cluster dynamics, including cluster-cluster merger and infall.

We present the MIR and other ancillary data used in this study in Section 2. In Section 3, we describe how MIR properties (colors) reflect the properties of A2255 member galaxies, and define the MIR diagnostics to study galaxies of different levels of MIR-excess. Using MIR diagnostics, we derive the star formation rates (SFRs) of individual galaxies and the entire cluster in Section 4. In Section 5, we discuss the relation between the environmental parameters and the MIR-excess galaxies.
Table 1

Observational Parameters of A2255 Data

\begin{tabular}{lcccc}
\hline \hline Filter & $\begin{array}{c}\lambda_{\text {eff }} \\
(\mu \mathrm{m})\end{array}$ & $\begin{array}{c}t_{\text {int }^{\mathrm{a}}} \\
(\mathrm{s})\end{array}$ & $\begin{array}{c}5 \sigma \text { Flux Limit } \\
(\mu \mathrm{Jy})\end{array}$ & $\begin{array}{c}\text { FWHM } \\
\left({ }^{\prime \prime}\right)\end{array}$ \\
\hline$N 3$ & 3.2 & 133.2 & 25 & 4.1 \\
$N 4$ & 4.1 & 133.2 & 30 & 4.0 \\
$S 7$ & 7.2 & 147.3 & 65 & 4.9 \\
$S 11$ & 10.4 & 147.3 & 80 & 5.3 \\
$L 15$ & 15.9 & 147.3 & 150 & 5.4 \\
$L 24$ & 23.0 & 147.3 & 400 & 6.3 \\
\hline
\end{tabular}

Notes.

a Total integration time per pixel.

b $5 \sigma$ flux limits are measured within an aperture of $2 \times$ FWHM diameter in each band image.

More detailed discussion suggesting the scenario of clusterscale merging in A2255 follows in Sections 6 and 7. Throughout this paper, we use a cosmology with $\Omega_{M}=0.3, \Omega_{\Lambda}=0.7$, and $H_{0}=70 \mathrm{~km} \mathrm{~s}^{-1} \mathrm{Mpc}^{-1}$ (e.g., Im et al. 1997). All the magnitudes are given in the $\mathrm{AB}$ system.

\section{DATA}

\subsection{AKARI Observation and Data Reduction}

CLEVL (CLusters of galaxies EVoLution studies) is one of the Mission Programs of the AKARI IR telescope (Murakami et al. 2007) designed to understand the formation and the evolution of galaxies in cluster environments. The program is divided into three major components according to the redshifts of the targets-low-redshift, intermediate-redshift, and highredshift clusters of galaxies. A2255 is one of the target galaxy clusters for the low-redshift CLEVL program (Im et al. 2008; Lee et al. 2009).

A2255 is observed by the AKARI InfraRed Camera (IRC; Onaka et al. 2007) over eight fields (Figure 1), with each field covering an area of $10 \times 10 \mathrm{arcmin}^{2}$. The IRC consists of three cameras - the NIR band camera [N2,N3,N4], the MIR$\mathrm{S}$ band camera [S7, $S 9 \mathrm{~W}, S 11]$, and the MIR-L band camera [L15, $L 18 W, L 24]$, where the number next to each alphabet denotes the central wavelength of the filter. Among these, the NIR and MIR-S band cameras are placed to observe the same field of view (FOV) on the sky, while the MIR-L band camera points to a different field separated by $\sim 10$ arcmin from the NIR and MIR-S FOV. The wide FOV $\left(10 \times 10 \operatorname{arcmin}^{2}\right)$ and the continuous wavelength coverage at 3-24 $\mu \mathrm{m}$ are two main advantages of $A K A R I$ with respect to Spitzer, in terms of the study of galaxy clusters. For the CLEVL low-redshift cluster programs, we used the IRC02 Astronomical Observation Template (AOT; see AKARI Observer's Manual version $1.2^{8}$ ) mode. This AOT takes moderate-length exposure by using two filters for each camera. The filter composition we selected is [N3, N4, S7, S11, L15 \& L24]. With this observational design, we obtain all-filter coverage for the central four fields $\left(\sim 400 \operatorname{arcmin}^{2}\right)$. Four fields in the south are covered only in $L 15$ and $L 24$ (dashed line in Figure 1), while four fields in the north are covered in $N 3 / N 4 / S 7 / S 11$ (solid line in Figure 1). Thus, the final data coverage with at least one $A K A R I$ filter is $1200 \operatorname{arcmin}^{2}$. The on-source exposure time for each filter is roughly $\sim 140 \mathrm{~s}$. The summary of the observation and the characteristics of the reduced images are specified in Table 1.

\footnotetext{
8 http://www.ir.isas.jaxa.jp/ASTRO-F/Observation/ObsMan/akobsman12.pdf
} 


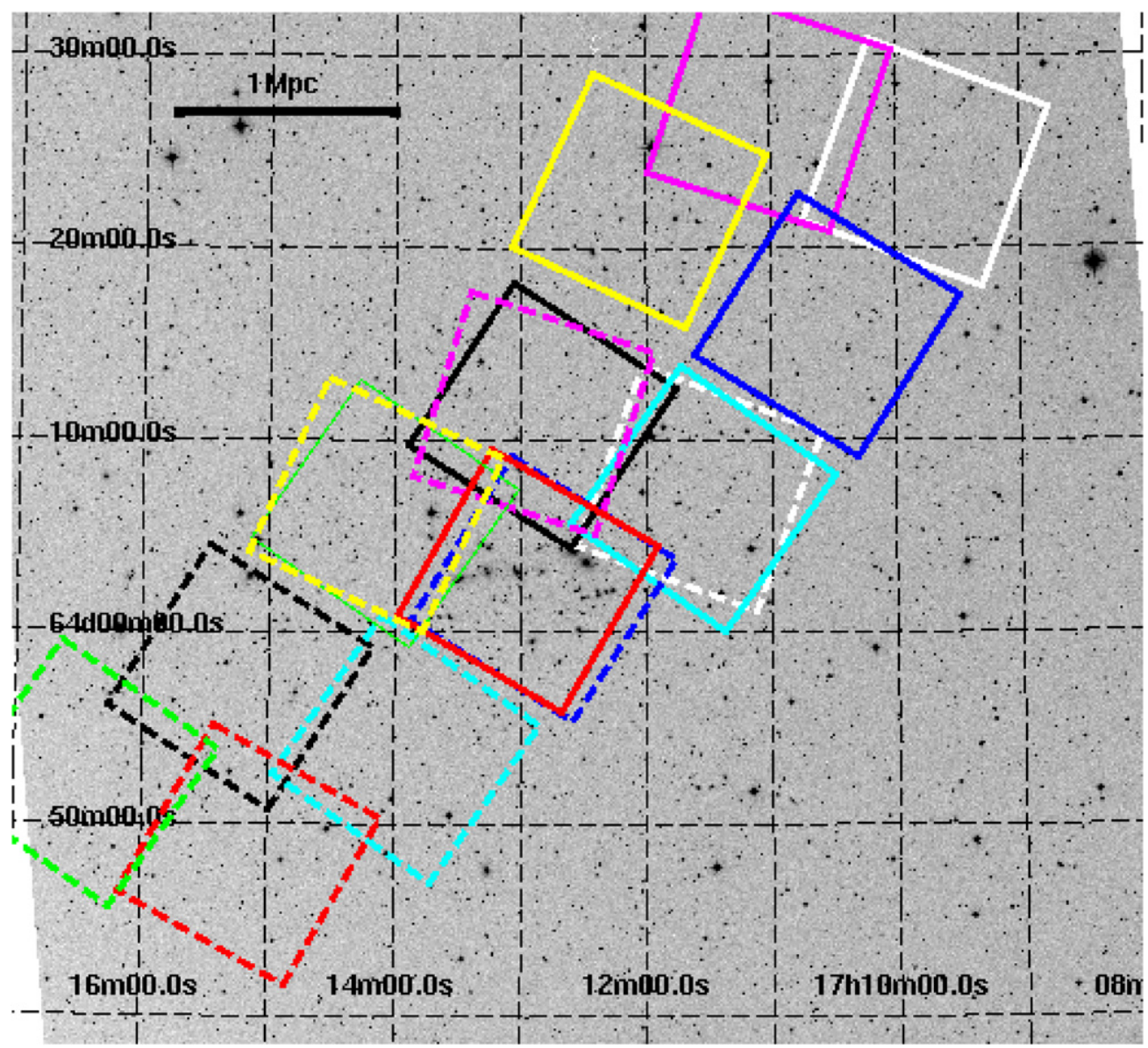

Figure 1. Field of views observed by AKARI overlaid on the DSS optical image of A2255. In total, twelve $10 \times 10 \operatorname{arcmin}^{2}$ fields are observed, among which a pair of fields marked with the same color is observed simultaneously with the MIR-L and NIR/MIR-S cameras. The dashed boxes indicate the MIR-L camera fields (L15 and L24), while the solid boxes indicate the regions observed by the NIR/MIR-S camera ( $N 3, N 4, S 7$, and $S 11)$.

(A color version of this figure is available in the online journal.)

The images are reduced using the IRC pipeline version $070104^{9}$ (provided as a form of the IRAF external package). The IRC pipeline consists of sky subtraction, astrometric calibration, and final coaddition of individual frames. For the MIR-L images whose astrometric calibration within the pipeline is relatively poor, we derive an astrometric solution using the IRAF task ccmap using Two Micron All Sky Survey sources or $S 7$ images as a reference. The full-width-at-half-maximum (FWHM) of the reduced images is between $44^{\prime \prime} 0-66^{\prime \prime} 0$, depending on the filter. The astrometric accuracy is $1^{\prime \prime}-2^{\prime \prime}$ rms for $N 3 / N 4 / S 7 / S 11$ images and $3^{\prime \prime}-4^{\prime \prime}$ rms for $L 15 / L 24$ images.

To measure the MIR fluxes, we use SExtractor (Bertin \& Arnouts 1996). In order to include all objects detected in at least one band, we make a reference image for source detection by combining images of different bands: for the photometry of $N 3$ through $S 11$ images, we use an $N 3 / N 4 / S 7 / S 11$ combined image as a reference image. Since the sensitivities in the $L 15$ and $L 24$ images are relatively poor compared to the case of $N 3-S 11$, we only combine L15 and L24 images to make a reference image for the photometry in $L 15 / L 24$ bands. We perform dualmode photometry using the reference images, with SExtractor configuration of DETECT_THRESH $=3.0$, DETECT_MINAREA = 2.0, and BACKGROUND mesh size of 32 pixels. As a measure of the total flux of each galaxy, we use FLUX_AUTO from the

\footnotetext{
9 http://www.ir.isas.jaxa.jp/ASTRO-F/Observation/DataReduction/IRC/ software/irc20070104.tgz
}

SExtractor output, i.e., the flux within a Kron elliptical aperture. The fluxes with a unit of ADU are converted to $f_{v}$ in $\mu \mathrm{Jy}$, using the IRC flux calibration table in the AKARI IRC data users' manual version $1.4(08 / 06 / 03 \text {; Lorente et al. 2007) })^{10}$. The "dual-mode" photometry we used in this paper is consistent with "single-mode" photometry, which is photometry performed on each image independently. The difference between dual-mode photometry and single-mode photometry is at most $<5 \%$ for each object.

\subsection{Cluster Member Identification}

After the construction of the AKARI band-merged catalog, we matched the $A K A R I$ photometry catalog with catalogs of previously known cluster member galaxies. In order to identify the membership for A2255, we use the spectroscopic redshifts from the SDSS DR2 (Abazajian et al. 2004) and BATC photometric redshifts by Yuan et al. (2003). Yuan et al. (2003) constructed a cluster member catalog that consists of 214 spectroscopic members and 313 photometric members out to $\sim 3 \mathrm{Mpc}$ from the cluster center. Since all the AKARI sources fall within the coverage of this catalog, we use the Yuan et al. (2003) cluster member catalog (hereafter the Y03 catalog) as a basis of our analysis in the following sections.

\footnotetext{
${ }^{10} \mathrm{http}: / /$ www.ir.isas.jaxa.jp/ASTRO-F/Observation/IDUM/ IRC_IDUM_1.4.pdf
} 
Table 2

AKARI IRC 3-24 $\mu \mathrm{m}$ and Spitzer MIPS 24/70 $\mu \mathrm{m}$ Photometry of A2255 Member Galaxies

\begin{tabular}{|c|c|c|c|c|c|c|c|c|c|}
\hline $\mathrm{ID}^{\mathrm{a}}$ & Redshift $^{\mathrm{b}}$ & $N 3^{\mathrm{c}}$ & $N 4$ & $S 7$ & $S 11$ & $L 15$ & $L 24$ & $24 \mu \mathrm{m}$ & $70 \mu \mathrm{m}$ \\
\hline A2255_S171036.20+642003.0 & 0.077836 & 16.27 & 16.64 & 14.91 & 14.80 & -1.00 & -1.00 & 14.36 & 10.41 \\
\hline A2255_S171117.90+640800.0 & 0.082396 & 16.26 & 16.87 & 16.09 & 15.60 & 15.90 & 15.44 & 15.51 & 13.33 \\
\hline A2255_S171226.40+640456.0 & 0.079492 & 16.80 & 17.50 & 18.11 & 99.00 & 18.56 & 99.00 & 99.00 & 99.00 \\
\hline A2255_S171234.10+640550.0 & 0.076180 & 17.84 & 18.40 & 16.76 & 16.31 & 16.02 & 14.72 & 14.77 & 11.64 \\
\hline A2255_S171236.10+640508.0 & 0.082686 & 16.96 & 17.62 & 18.35 & 18.51 & 17.84 & 99.00 & 99.00 & 99.00 \\
\hline A2255_P171240.34+640443.1 & 0.083 & 17.25 & 17.93 & 17.74 & 17.14 & 17.78 & 99.00 & 17.96 & 12.88 \\
\hline A2255_P171247.18+635625.0 & 0.079 & 17.22 & 17.81 & -1.00 & -1.00 & 99.00 & 17.58 & 17.02 & 99.00 \\
\hline A2255_P171257.56+641028.2 & 0.087 & 18.09 & 18.63 & 17.41 & 16.72 & 17.28 & 16.97 & 16.17 & 13.15 \\
\hline A2255_P171335.98+640747.1 & 0.090 & 16.97 & 17.61 & 17.41 & 16.88 & 17.09 & 16.76 & 16.86 & 99.00 \\
\hline A2255_S171343.50+640502.0 & 0.090206 & 99.00 & 99.00 & 15.53 & 15.28 & 15.66 & 15.53 & 15.41 & 11.65 \\
\hline A2255_S171352.00+640710.0 & 0.082871 & 17.33 & 17.82 & 16.04 & 15.74 & 15.74 & 15.45 & 15.23 & 12.93 \\
\hline A2255_P171406.11+641019.9 & 0.089 & 17.57 & 18.20 & 17.38 & 17.08 & 17.55 & 17.33 & 17.31 & 99.00 \\
\hline A2255_P171452.86+640649.3 & 0.079 & 17.29 & 17.94 & 18.60 & 99.00 & 99.00 & 99.00 & 99.00 & 99.00 \\
\hline A2255_S171602.10+635729.0 & 0.079818 & -1.00 & -1.00 & -1.00 & -1.00 & 16.42 & 16.03 & 99.00 & 99.00 \\
\hline A2255_P171602.36+635755.0 & 0.079000 & -1.00 & -1.00 & -1.00 & -1.00 & 17.40 & 17.18 & 99.00 & 99.00 \\
\hline
\end{tabular}

Notes.

a ID of galaxy represents whether the galaxy is a spectroscopic member (“A2255_S”) or a photometric member (“A2255_P”), and the coordinates of the galaxy in sexagesimal format in R.A./decl.

b Redshifts of the spectroscopically selected members ("S") are from SDSS DR2 (Abazajian et al. 2004), while redshifts of the photometrically selected members ("P") are from Yuan et al. (2003).

${ }^{c}$ The unit of $N 3, N 4, S 7, S 11, L 15, L 24$, MIPS $24 \mu \mathrm{m}$ and $70 \mu \mathrm{m}$ values are AB magnitudes. The magnitudes represent total magnitudes of the galaxies. The value 99.00 indicates non-detection (below the detection limit-see the text for details), and - 1.00 indicates that the source is either out of the field of view in the observation, blended, etc.

(This table is available in its entirety in a machine-readable form in the online journal. A portion is shown here for guidance regarding its form and content.)

The SDSS spectroscopic targets are selected down to $r \lesssim$ 17.77 mag after correcting for Galactic extinction (Strauss et al. 2002), therefore all members with spectroscopic redshifts are brighter than $17.77 \mathrm{mag}$ in the $r$ band. The completeness of the cluster member selection with spectroscopic redshifts at this magnitude range is on average $\sim 85 \%$, although it depends on the magnitudes and the clustercentric distances of the galaxies (see Figure 1 in Park \& Hwang 2009). The addition of cluster members with photometric redshifts corrects this incompleteness. Note that the number of member galaxies with photometric redshifts brighter than $r=17.77$ mag is 40 . The magnitude limit for the BATC photometry is $\sim 20$ mag in each BATC filter, and the BATC photometric redshift identification is nearly complete for galaxies brighter than $V<19$ mag (Yuan et al. 2003). This magnitude corresponds to $r<18.6-18.9$ mag for different types of galaxies (c.f. galaxy colors in various photometric band systems; Fukugita et al. 1995). Therefore, the cluster member selection is complete down to $r<18.6-18.9 \mathrm{mag}$ in terms of both the redshift identification and the member identification. Above this limit, there are more than $\sim 100$ member galaxies with $18.9 \mathrm{mag}<r<20 \mathrm{mag}$. We expect a significant incompleteness in cluster member selection for this magnitude range. Although it is difficult to estimate the exact incompleteness at this faint end, this sample incompleteness would not cause much problem considering the relatively high MIR detection limit compared to the optical limit. We describe the effect of the incompleteness on our analysis in more detail in Section 4 (also, please refer to Figure 8). The redshift cut for A2255 member galaxies is $0.068<z<0.090$, and the median redshift of A2255 member galaxies is $\langle z\rangle=0.081$.

The matching between the Y03 catalog and the AKARI IR source catalog is done using a matching radius of $5^{\prime \prime}$ (roughly $\sim 1.5 \times$ FWHM radius of the $A K A R I \mathrm{~N} 3$ image, close to FWHM in other bands). We checked the optical and $A K A R I$ images of individual member galaxy to confirm that the matched object is not blended or mis-matched with neighboring sources. Most $(\sim 95 \%)$ of the member galaxies are not blended in MIR. For $5 \%$ of galaxies that are suspected to be blended in MIR, we assign the "-1.00" value in the final photometry table (Table 2). Galaxies outside the survey coverage are also assigned " -1.00 ," and these are not included in the following analysis. If the galaxy is within the survey coverage and has the flux below the detection limit, we assign "99.00." We identify 122 spectroscopically confirmed member galaxies and 170 member galaxies selected by photometric redshifts ( 292 in total) in the Y03 catalog which have AKARI MIR counterparts, over $\sim 1200 \mathrm{arcmin}^{2}$ of the $A K A R I$ observation.

\subsection{Spitzer MIPS Photometry}

A2255 is observed by Spitzer MIPS $24 \mu \mathrm{m}$ and $70 \mu \mathrm{m}$ at $5 \sigma$ flux limits of $250 \mu \mathrm{Jy}$ and $5 \mathrm{mJy}$, respectively (PID 40562, PI: G. Rieke). Since the coverage of MIPS $24 \mu \mathrm{m}$ is $\sim 1500 \mathrm{arcmin}^{2}$, and it includes the region not covered by $A K A R I$ (see Figure 11(b) for comparison of the AKARI IRC and the Spitzer MIPS coverages), we derive the MIPS 24/70 $\mu \mathrm{m}$ photometry for cluster member galaxies within the Spitzer MIPS coverage. The inclusion of $24 / 70 \mu \mathrm{m}$-detected galaxies increases the number of member galaxies detected in MIR.

We run SExtractor over pbcd (post-Basic Calibrated Data) MIPS images to measure $24 / 70 \mu \mathrm{m}$ fluxes. We use FLUX_AUTO of each object as total flux like we did in the case of the AKARI IRC bands. The FLUX_AUTO is measured at the previously defined optical coordinates of galaxies (i.e., the Y03 catalog) using ASSOC parameters. All AKARI-Y03 matched members have either $24 / 70 \mu \mathrm{m}$ flux or upper limits, and there are 134 additional member galaxies in the Y03 catalog that lie within Spitzer MIPS coverage and outside the AKARI coverage.

In Table 2, we present MIR photometry of A2255 member galaxies from the AKARI and Spitzer observations. The table contains photometry for 426 member galaxies from the Y03 
catalog: 292 galaxies within AKARI IRC fields, 134 galaxies within Spitzer MIPS coverage but outside the AKARI IRC coverage. The columns in the table are galaxy id (with coordinate information), spectroscopic or photometric redshift, $A K A R I$ IRC flux $(3,4,7,11,15$, and $24 \mu \mathrm{m})$, and MIPS 24 and $70 \mu \mathrm{m}$ flux. As mentioned in Section 2.2, the value of -1.00 in the magnitude column indicates that the object is not covered by the corresponding filter, and 99.00 indicates that the flux of the object is below the detection limit.

\subsection{Multi-wavelength Ancillary Data}

In this section, we describe other wavelength data used in this study: optical imaging and spectroscopy, UV imaging, radio, and X-ray imaging.

In the optical wavelengths, we use the ugriz SDSS photometry as well as the 13-band photometry (from UV to $i$ band) in Yuan et al. (2003). These optical photometric points are used to construct the spectral energy distributions (SEDs) of cluster member galaxies (see Section 3.3 for details). We also use the spectroscopic redshift from the SDSS database for membership identification. The line equivalent widths and the stellar metallicities are taken from the SDSS-MPA catalog ${ }^{11}$ and from Gallazzi et al. (2005).

We also used the UV flux information which provides another measure of SFRs (see Section 4.1 for details). We queried the Galaxy Evolution Explorer (GALEX) source catalog from the all sky survey and the nearby galaxies survey ${ }^{12}$ (tile UVE_A2255) over $\sim 1 \mathrm{deg}^{2}$ of A2255. We found 313 matches within $10^{\prime \prime}$ from the optical coordinates of A2255 member galaxies within the MIR coverage (Note that the total number of member galaxies with the MIR data is 426; Section 2.3). For 76 galaxies that are used in the comparison between UV- and IR-derived SFRs (Section 4.1), we visually inspected the UV images to confirm whether the cross-identification is correct and the UV flux is not contaminated by nearby sources. The depths in FUV (1500 $\AA$ ) and NUV $(2300 \AA)$ wavelengths are roughly $\sim 20 \mu \mathrm{Jy}$ at the $5 \sigma$ flux limit.

The radio flux is also used in the comparison between the different SFR indicators (Section 4.1). We use the $1.4 \mathrm{GHz}$ radio source catalog of Miller \& Owen (2003) and cross-identify the sources with IR-detected cluster members using a matching radius of $5^{\prime \prime}$. The number of matched members within MIR FOV is 33. Among these, 20 galaxies are classified as starforming galaxies, 3 as Seyfert galaxies, and the remaining 10 galaxies as AGN candidates with old stellar population (Miller \& Owen 2003; the classification is based on the optical spectra of galaxies).

Finally, we use the X-ray point source catalog obtained with Chandra (Davis et al. 2003) and cross-identify eight sources using a matching radius of $5^{\prime \prime}$. These sources overlap with radio sources (Miller \& Owen 2003) described above. We present a separate section about AGNs in Section 3.5.

\section{MIR PHOTOMETRIC PROPERTIES OF A2255 GALAXIES AND MIR-EXCESS GALAXIES}

In this section, we explore the MIR properties of galaxies in A2255 and provide a classification method based on the excess in the MIR emission in order to facilitate the study of environmental dependence of the galaxy evolution in the cluster.

\footnotetext{
11 http://www.mpa-garching.mpg.de/SDSS/DR4/

12 http://galex.stsci.edu/GR4/
}

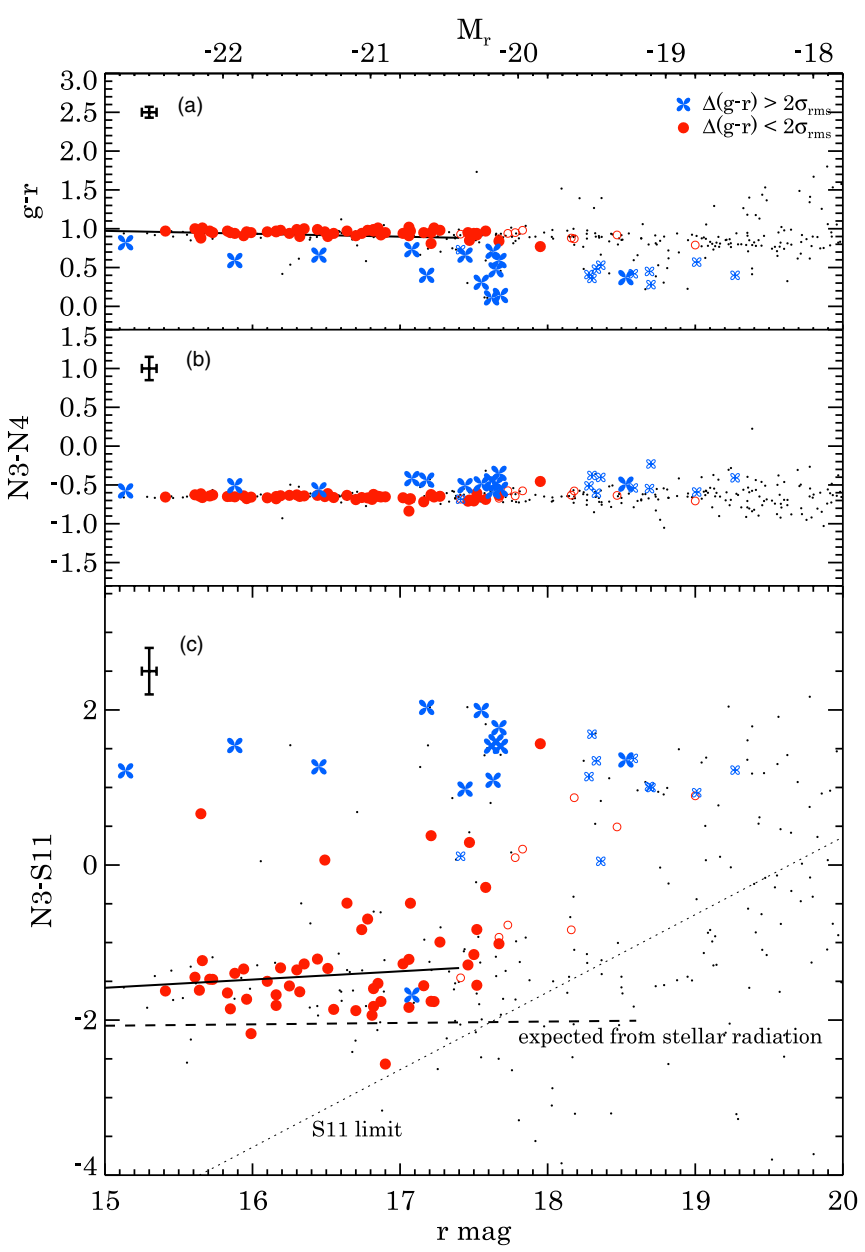

Figure 2. Color-magnitude diagram of galaxies in the A2255 field at different wavelengths. From top (a) to bottom (c), the $y$-axis of the color-magnitude diagram is $(g-r),(N 3-N 4)$, and $(N 3-S 11)$. The $x$-axis is identical to $r$-magnitudes. The colored points are galaxies detected in $S 11\left(f_{S 11}>80 \mu \mathrm{Jy}\right)$ : red circles indicate galaxies lying at optical red sequence, blue clovers indicate galaxies outside the red sequence. Filled symbols are member galaxies with spectroscopic redshifts and open symbols are galaxies with photometric redshifts. The small dots indicate all non-star objects in the observed field. The length of the $y$-axis is proportional to the $y$-range for each panel. The error bar in top left of each panel indicates typical errors in colors and magnitudes. The solid line in panels (a) and (c) is the linearly fitted color-magnitude relation (Equations (1) and (2)), and the dashed line in panel (c) indicates the expected $N 3-S 11$ colors from stellar radiation only. The dotted line in panel (c) is an $N 3-S 11$ limit produced due to the $S 11$ limit of $f_{S 11}>80 \mu \mathrm{Jy}$.

(A color version of this figure is available in the online journal.)

\subsection{Color-Magnitude Relation}

It is well known that cluster member galaxies show a clear red sequence in their color-magnitude relation defined by elliptical galaxies, reflecting the similar age and/or metallicity of galaxies (e.g., Gladders et al. 1998). We investigate the color-magnitude relation of A2255 member galaxies at different wavelengths - optical, NIR, and MIR. Figure 2 represents the color-magnitude diagram of A2255 member galaxies in $g-r$, $N 3-N 4$, and $N 3-S 11$, from top to bottom.

In the optical and NIR (Figures 2(a) and (b)), there is a tight sequence of galaxies in the color-magnitude diagram. In the optical color-magnitude diagram, a tight sequence made by bright, passively evolving member galaxies can be seen. The same sequence appears in $r$ versus $N 3-N 4$ color-magnitude relation, but in a reversed way that the brighter galaxies 
have the bluer $N 3-N 4$ colors (Figure 2(b)). We define the "red sequence" in the optical color-magnitude diagram to divide the member galaxies into two groups: (1) red-sequence galaxies and (2) non red-sequence galaxies. All the member galaxies with spectroscopic redshifts in the non-red-sequence category show nebular emission lines indicative of ongoing star formation, thus non-red-sequence galaxies are thought to be star-forming galaxies while red-sequence galaxies are not. The color-magnitude relation is derived using a linear fit to $r$ versus the $g-r$ relation for $r<17.5$ mag galaxies (see the solid line in Figure 2(a)), by excluding the outliers iteratively based on the bi-weight estimator. The fitted color-magnitude relation is as follows:

$$
g-r=-0.037 \times r+1.53 .
$$

The standard deviation of residuals to this fit is $\sigma_{\mathrm{rms}}=0.08$ mag, indicating the tightness of the optical red sequence. This value for scatter includes the photometric errors, and the slope in color-magnitude relation is consistent with that derived in other studies (e.g., Gallazzi et al. 2006). We consider galaxies to belong to the red sequence if they lie within $\Delta(g-r)<2 \sigma_{\text {rms }}$ from the red sequence, where $\Delta(g-r)$ is an offset of color from the fitted relation. In Figure 2, circles indicate optical red-sequence galaxies and clovers indicate non red-sequence galaxies. While the optical red sequence is produced by galaxies with similar ages and metallicities, the same objects form an NIR "blue" sequence since we are sampling the Rayleigh-Jeans tail of the black body radiation with the $N 3-N 4$ colors (e.g., Lacy et al. 2004; Stern et al. 2005). In NIR, the non-red-sequence galaxies in the optical have the redder $N 3-N 4$ colors than the $N 3-N 4$ blue sequence-possibly due to the dust emission arising from star formation.

In contrast, the tight sequence deteriorates in the color-magnitude relation in MIR, e.g., in the $r$ versus $N 3-S 11$ color-magnitude diagram (Figure 2(c)). The spread in N3-S11 colors is much larger than those in optical or NIR, even if the outliers (clovers) from the optical red sequence are excluded. The linear relation derived from the $r$ versus $N 3-S 11$ diagram (solid line in Figure 2(c)) using the same method as the optical is

$$
N 3-S 11=0.106 \times r-3.18 .
$$

The scatter around this relation is $\sigma_{\mathrm{rms}}=0.44 \mathrm{mag}$, roughly six times larger than that in the optical. The large scatter of $\sigma_{\text {rms }}=0.44 \mathrm{mag}$ in the MIR blue sequence cannot be fully accounted for even if a maximum photometric error of $\sim 0.3 \mathrm{mag}$ in the $S 11$ band is assumed.

In addition to the large scatter, there are two interesting characteristics in the $N 3-S 11$ color-magnitude diagram. The first is that there is a weak "blue" sequence of galaxies, although the scatter is very large as described above. Contrary to the case of optical color-magnitude relation, the brighter galaxies have bluer MIR color. The second is that most of the galaxies detected in the $S 11$ band show redder $N 3-S 11$ colors than expected from stellar radiation alone $(N 3-S 11 \sim-2.0$; dashed line in Figure 2(c)). The value of $N 3-S 11 \sim-2.0$ is calculated using a model of Piovan et al. (2003), where only stellar photospheric emission is considered-i.e., no dust continuum is taken into account. Not only galaxies that are outliers of $N 3-S 11$ blue sequence, but also galaxies in the blue sequence are considered to have "MIR-excess" compared to the stellar continuum. The possible origins of the MIR-excess in these galaxies are the dust emission related to the star formation, AGN activity, and the circumstellar dust shells around AGB stars (e.g., Ko et al.
2009), etc. We investigate these possibilities by comparing MIR colors with galaxy model expectations in the following section.

\subsection{Color-Color Diagram}

In order to understand origins of the MIR excess in more detail, we present an MIR color-color diagram of A2255 member galaxies in Figure 3(a). The MIR colors used here are $N 3-S 7$ versus $N 3-S 11$, and the expected colors from various model galaxy templates are overplotted. The overplotted color tracks are calculated using models of elliptical galaxies with different ages and metallicities which include the dust emission from circumstellar dust around AGB stars (Piovan et al. 2003) or SED templates of local star-forming galaxies and IR luminous galaxies (Chary \& Elbaz 2001).

The plot shows that galaxies in the $N 3-S 11$ blue sequence $(\langle N 3-S 11\rangle \sim-1.7)$ are likely to be passively evolving galaxies with stellar population age around $10 \mathrm{Gyr}$ or larger. The reddest in both MIR colors $(N 3-S 11>0.2 \mathrm{mag}$ and $N 3-S 7>0 \mathrm{mag})$ are star-forming galaxies. Galaxies between two populations are dominated by young, passively evolving galaxies with stellar ages between 1 and $10 \mathrm{Gyr}$, yet these could also be galaxies with a small amount of star formation.

Based on the color tracks of model galaxy templates (Figure 3(a)), we classify MIR-excess galaxies into three classes according to the amount of MIR excess. The reddest galaxies, having N3 $-S 11>0.2 \mathrm{mag}$ are defined as "strong MIRexcess," where the MIR color corresponds to that of an actively star-forming galaxy calculated using SED templates of Chary \& Elbaz (2001). Galaxies at the $N 3-S 11$ blue sequence are defined as "weak MIR-excess" galaxies $(N 3-S 11<-1.2 \mathrm{mag})$, which have MIR colors of passively evolving galaxies with old ages. The objects between the two populations are tagged as "intermediate MIR-excess" galaxies whose MIR colors can have multiple origins such as circumstellar dust emission from intermediate age stars, residual star formation, and AGN activity. These three classifications based on the MIR color allow us to investigate galaxy populations at different evolutionary stages. The environmental dependence of galaxies is discussed in the following sections based on these classifications.

These MIR-excess terms are defined using N3 - S11 colors and thus limited by $N 3$ and $S 11$ coverage (Figure 1) and depth. Therefore, we develop another criteria to define MIR excess using $24 \mu \mathrm{m}$ flux from Spitzer MIPS, which covers a larger area than the $S 11$ coverage. Figure 3(b) shows the correlation between $N 3-S 11$ and $\operatorname{mag}(z)-\operatorname{mag}(24 \mu \mathrm{m})$ for the observed galaxies, in addition to the expected model colors. Two MIR colors, $N 3-S 11$ and $\operatorname{mag}(z)-\operatorname{mag}(24 \mu \mathrm{m})$ correlate reasonably well, and the locations of model tracks are consistent with the case of $N 3-S 7$ versus $N 3-$ $S 11$. Therefore we use $\operatorname{mag}(z)-\operatorname{mag}(24 \mu \mathrm{m})$ to define MIRexcess galaxies in addition to $N 3-S 11$. The corresponding criteria for strong/intermediate/weak MIR excess are, $\operatorname{mag}(z)-\operatorname{mag}(24 \mu \mathrm{m})>-0.5,-2.0<\operatorname{mag}(z)-\operatorname{mag}(24 \mu \mathrm{m})$ $<-0.5$, and $-3.5<\operatorname{mag}(z)-\operatorname{mag}(24 \mu \mathrm{m})<-2.0$, respectively.

In Table 3, we compare the number of cluster member galaxies with or without MIR detection, and MIR excess. The fractions of MIR-excess galaxies among cluster members at the same $r$-band magnitudes are different for the AKARI $S 11$ and Spitzer $24 \mu \mathrm{m}$ fields, e.g., $74 \%$ for $S 11$ and $37 \%$ for $24 \mu \mathrm{m}$ at $r<17.5$. This difference is due to the shallower depth of the $24 \mu \mathrm{m}$ image compared to the $S 11$ image. As we showed in Figure 2(c), $S 11$ flux limits or $24 \mu \mathrm{m}$ flux limits place limits on the MIR color that can be considered as MIR excess. With $5 \sigma$ 

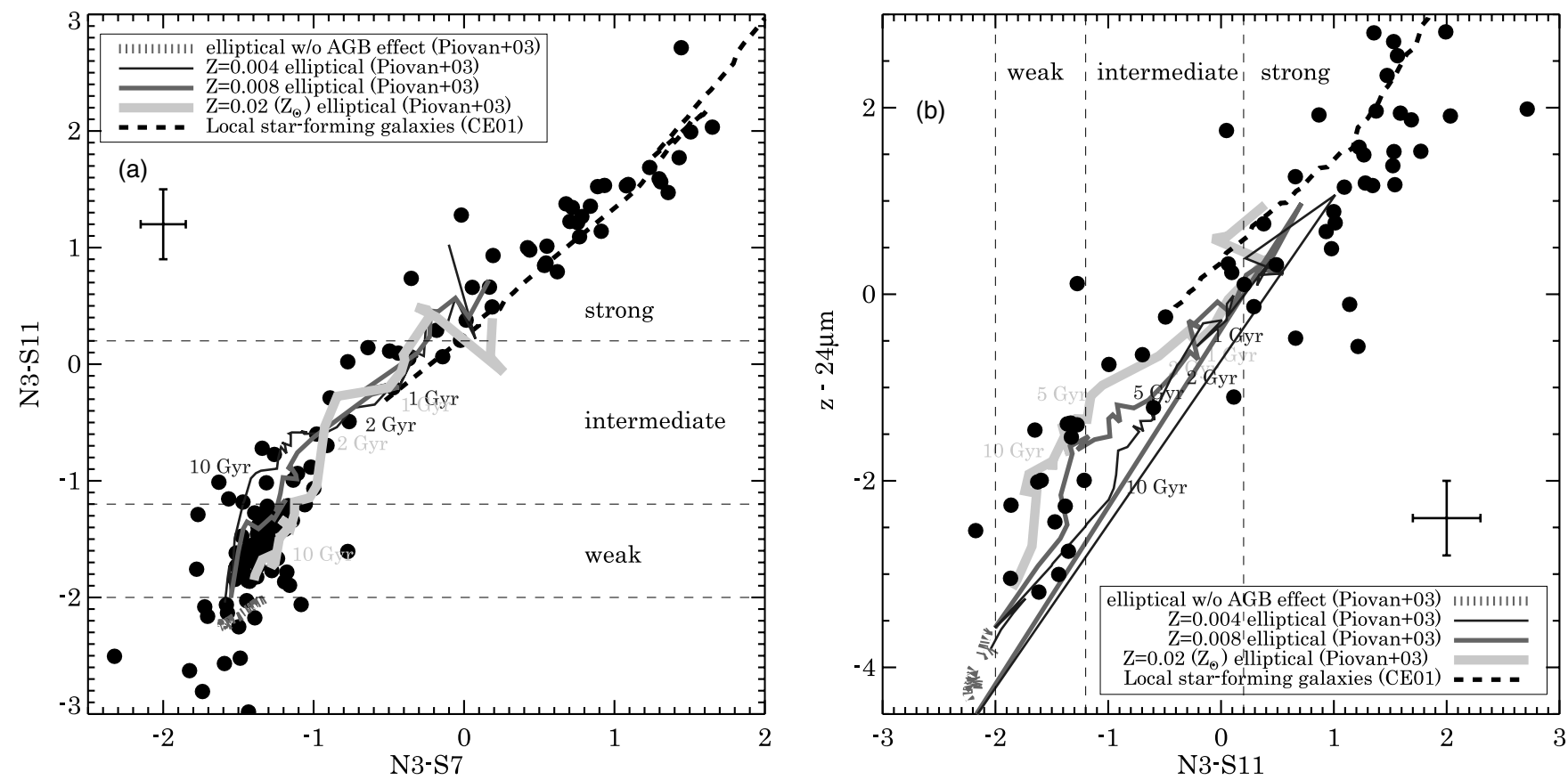

Figure 3. Left: $(N 3-S 7)$ vs. $(N 3-S 11)$ color-color plot of A2255 member galaxies. Overplotted lines are the expected tracks of local star-forming galaxies (Chary \& Elbaz 2001; thick dashed line), elliptical galaxies with different metallicities (Piovan et al. 2003; solid line). The metallicity increases from left $(Z=0.004)$ to right $(Z=0.02)$ in the $x$-axis, the age decreases as $(N 3-S 11)$ gets redder, and the total IR luminosity increases as $(N 3-S 7)$ and $(N 3-S 11)$ increase. There is an overlap between the star-forming galaxy track and elliptical galaxies' tracks around $(0,0)$. Filled circles are points from cluster member galaxies. The error bar indicates typical color errors produced by magnitude errors. Right: the criteria for MIR excess in terms of MIR colors $(N 3-S 11$ and $\operatorname{mag}(z)-\operatorname{mag}(24 \mu \mathrm{m}))$. As in (a), we use the $N 3-S 11$ color as a measure of MIR excess. Over the area where no $3 \mu \mathrm{m}$ or $11 \mu \mathrm{m}$ images are available, we use $\operatorname{mag}(z)-\operatorname{mag}(24 \mu \mathrm{m}) \operatorname{colors}$ instead of $N 3-S 11$ colors. Filled circles are points from cluster member galaxies with MIR-excess, while various lines are the expected relation from different models. Again, the error bar indicates typical color errors.

Table 3

Number of A2255 Member Galaxies

\begin{tabular}{|c|c|c|c|c|c|c|}
\hline \multirow[t]{2}{*}{ Description } & \multicolumn{3}{|c|}{$S 11$} & \multicolumn{3}{|c|}{$24 \mu \mathrm{m}$} \\
\hline & $r<17.5$ & $17.5<r<18.5$ & $r>18.5$ & $r<17.5$ & $17.5<r<18.5$ & $r>18.5$ \\
\hline Cluster member galaxies & 81 & 78 & 54 & 158 & 146 & 122 \\
\hline MIR detections & 63 & 26 & 13 & 59 & 38 & 22 \\
\hline Galaxies with MIR-excess & 60 & 24 & 13 & 59 & 38 & 22 \\
\hline Weak MIR-excess & 42 & 1 & $\ldots$ & 12 & $\ldots$ & $\ldots$ \\
\hline Intermediate MIR-excess & 9 & 8 & $\ldots$ & 20 & 3 & $\ldots$ \\
\hline Strong MIR-excess & 9 & 15 & 13 & 27 & 35 & 22 \\
\hline
\end{tabular}

Notes. Columns 1-3 indicate the number of A2255 member galaxies in AKARI/IRC S11 field of view, and Columns 4-6 indicate the number of galaxies in Spitzer/MIPS $24 \mu \mathrm{m}$ area coverage. MIR detections are defined as $f_{S 11}>80 \mu \mathrm{Jy}$ and $f_{24 \mu m}>250 \mu \mathrm{Jy} ; 5 \sigma$ flux limits as mentioned in Section 2. The criteria for weak/intermediate/strong MIR-excess galaxies are described in Section 3.2.

flux limit of $80 \mu \mathrm{Jy}$ for $S 11$ band, weak MIR excess galaxies are complete only at $r<17.5$, while intermediate and strong MIR-excess galaxies are complete down to $r \sim 18.5$ mag. On the other hand at $24 \mu \mathrm{m}$ with flux limit of $250 \mu \mathrm{Jy}$, the complete limit for weak MIR-excess galaxies is $r<15 \mathrm{mag}$, and the limit for intermediate MIR-excess galaxies is $r<16.5 \mathrm{mag}$. Since our survey consists of fields covered with different filters having different depths, we take this different MIR-excess fraction into account when discussing the spatial distribution of MIR-excess galaxies (Section 5).

Table 3 as well as Figure 2 shows that the fraction of MIRexcess galaxies is very high $(>70 \%$ at $r<17.5 \mathrm{mag}$, using $S 11)$, contrary to the general belief that there are little dust and gas among cluster member galaxies. Many of these MIR-excess galaxies are on the optical red sequence (weak and intermediate MIR-excess galaxies; see Figure 2(c)), so that it is essential to include AGB circumstellar dust emission when describe the
SED of red cluster galaxies from optical through MIR (Bressan et al. 2007). This is also consistent with the trend in other galaxy cluster, A2218, in which a significant MIR-excess is seen in fainter early-type member galaxies (Ko et al. 2009).

\subsection{Spectral Energy Distributions and Morphologies}

For the MIR-excess galaxies defined in Section 3.2, we calculate either total IR luminosity (i.e., IR-derived SFR) or stellar population age based on the SED fitting. The derivation of these quantities allows us to investigate star formation activities / quenching sequence and its relation to the environment in A2255. The SED fitting follows the procedure described in Shim et al. (2007) except that we include the photometric data from optical (SDSS ugriz) to MIR (AKARI IRC points and Spitzer MIPS $24 / 70 \mu \mathrm{m}$ points if available). The template library we used is IR galaxy templates with different IR luminosities (Chary \& Elbaz 2001), and the early-type galaxy templates from 


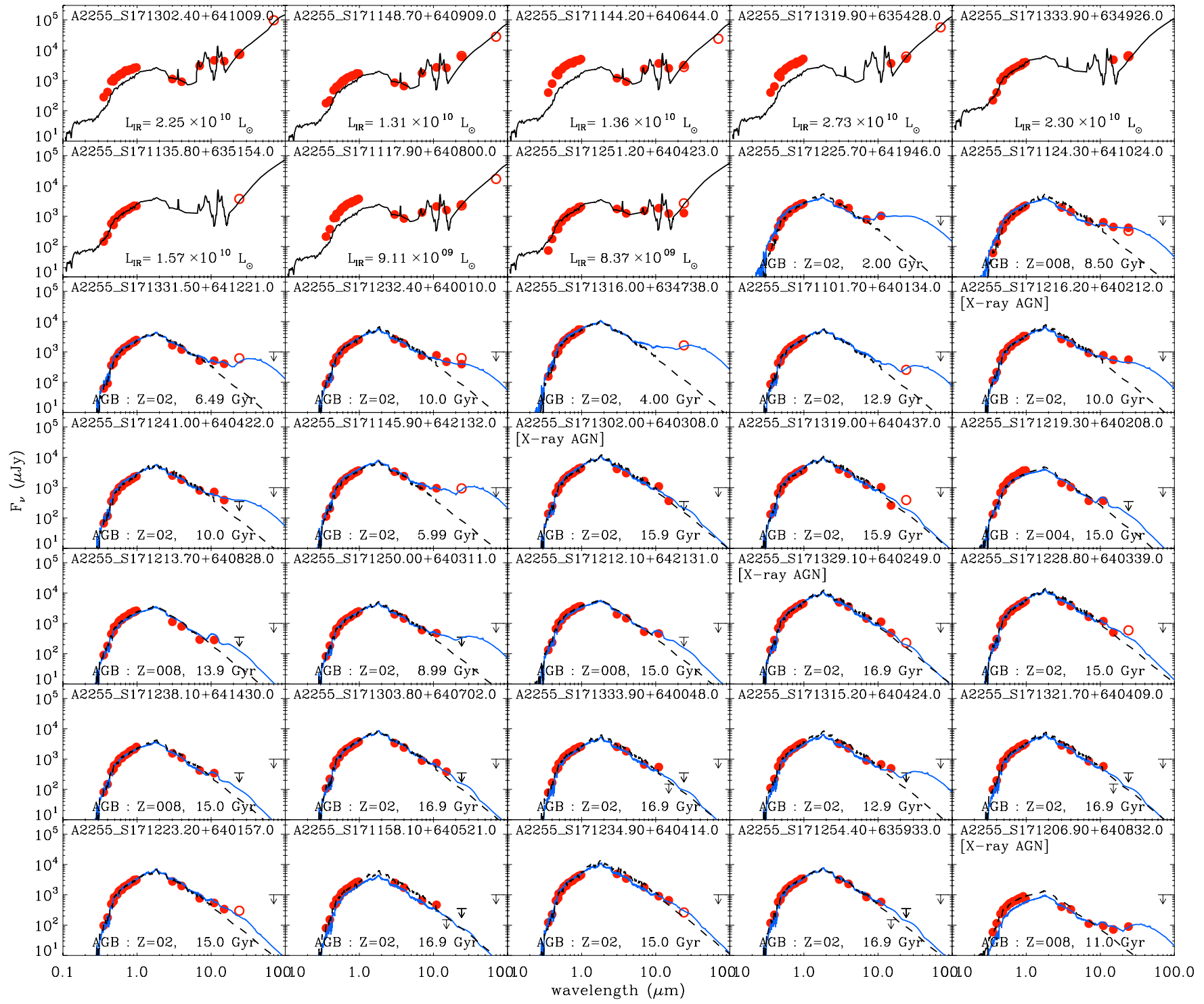

Figure 4. SEDs of 34 "optically" bright $(r<16.5 \mathrm{mag})$ galaxies in addition to one optically faint $(r \sim 17.0 \mathrm{mag})$ X-ray-selected AGN. The morphologies of these galaxies are also presented in Figure 5, with the same order. Filled circles indicate optical and AKARI IRC photometry data points, while open circles indicate MIPS $24 \mu \mathrm{m}$ and $70 \mu \mathrm{m}$ data points when available. In case we do not have detection despite the coverage, we mark flux upper limits with $5 \sigma$ with arrows. Overplotted lines represent the best-fit SEDs through the SED fitting using optical to MIR bands: the black solid lines indicate IR galaxy templates of Chary \& Elbaz (2001), blue solid lines indicate the early-type galaxy templates considering the effect of AGB dust (Piovan et al. 2003), and black dashed lines indicate early-type galaxy templates without AGB dust effect (Piovan et al. 2003). Note that for star-forming galaxies, we only used MIR (3-70 $\mu$ m) photometry data points in SED fitting since the optical-NIR part of the SEDs was arbitrarily defined in Chary \& Elbaz (2001).

(A color version of this figure is available in the online journal.)

Piovan et al. (2003). The IR galaxy templates are constructed through empirical interpolation between the observed local IR galaxies. As it is mentioned in Chary \& Elbaz (2001), the optical-NIR part of the SEDs are arbitrarily determined to match the IR versus optical luminosity ratio, and thus we used MIR (3-70 $\mu \mathrm{m})$ data points only to fit the IR SEDs. The earlytype galaxy templates are constructed considering the effect of circumstellar dust around AGB stars to the integrated spectrum in addition to the photospheric emission. The best-fit template is found using $\chi^{2}$ minimization between the model fluxes and the observed fluxes.

Figure 4 shows the examples of the SED fitting for 34 relatively bright galaxies in optical $\left(r<16.5 \mathrm{mag}\right.$, i.e., $M_{r}<$ $-21.0 \mathrm{mag}$ ). These SED panels are arranged in descending order of $N 3-S 11$ values. Since these galaxies are sufficiently bright and large, we also present their color-composite stamp images in Figure 5 as a guide to their morphologies. The images are color composites of SDSS $g$ (blue), $r$ (green), and $i$ (red) band inverse images. The size of each cutout is $30^{\prime \prime} \times 30^{\prime \prime}$, and the images are displayed in logarithmic scale. The objects in Figure 5 are aligned with the same order as Figure 4. At $r>16.5 \mathrm{mag}$, it becomes difficult to investigate the morphologies using the SDSS images unless they are sufficiently extended. The last object in Figures 4 and 5 does not fall in the magnitude cut for optically bright objects, yet the object is included as an example of member galaxies with known AGNs showing MIR-excess.

The MIR $(N 3-S 11)$ colors are marked in the lower right of each cutout image in Figure 5. The first seven galaxies are strong MIR-excess galaxies, and the eighth galaxy is an intermediate MIR-excess galaxy. These eight galaxies are 


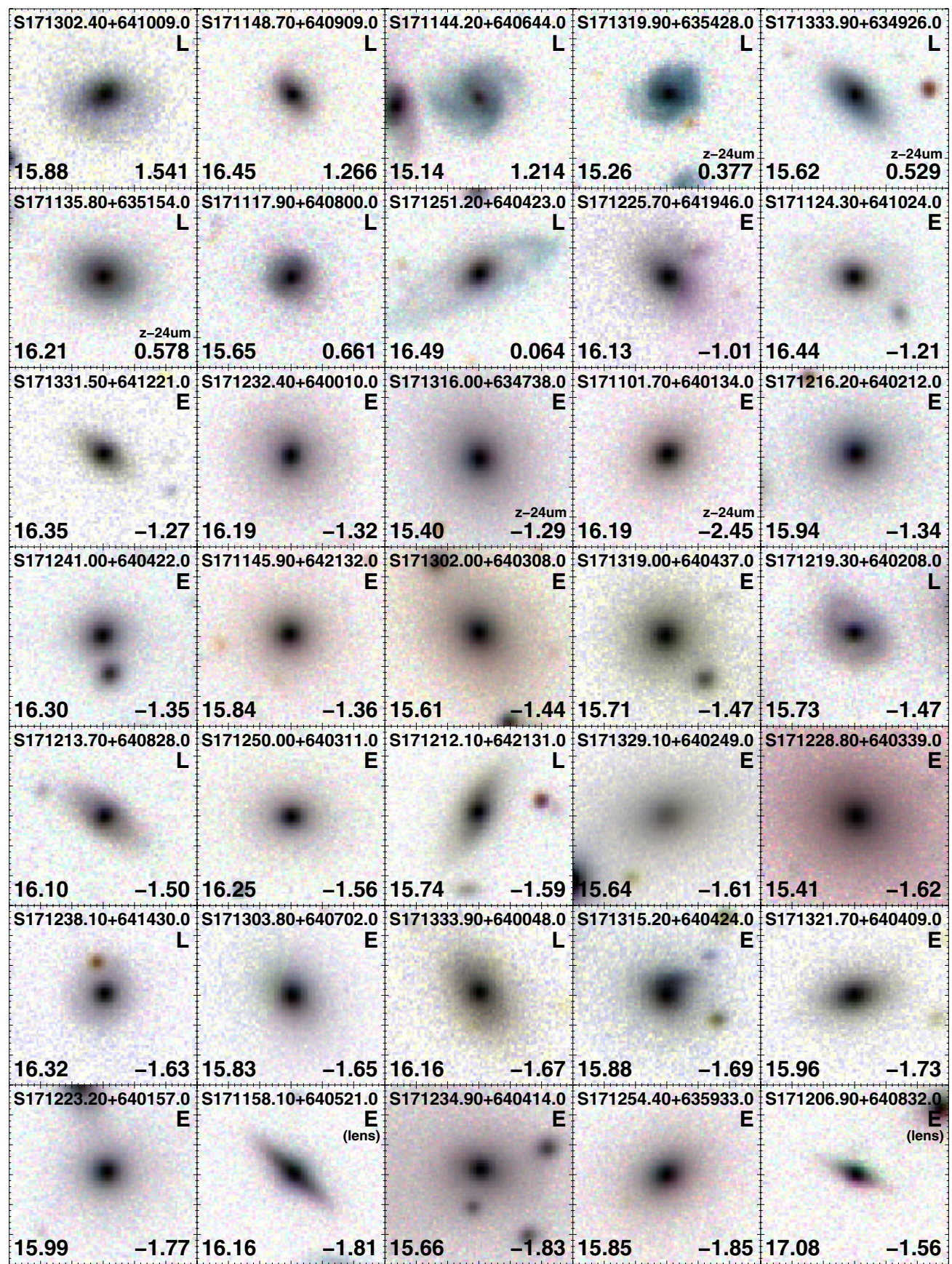

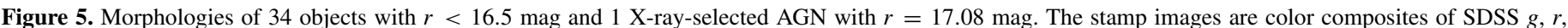

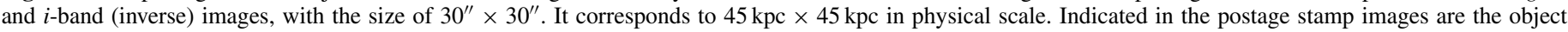

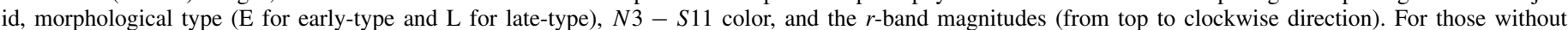

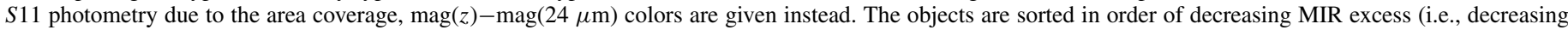
$N 3-S 11$ colors), except the last AGN object.

(A color version of this figure is available in the online journal.)

best-fitted with IR galaxy templates (Chary \& Elbaz 2001) and show late-type morphology or disky features. The ninth galaxy is an intermediate MIR-excess galaxy, best-fitted with an early-type galaxy template. All the remaining galaxies are weak MIR-excess galaxies, which are best-fitted by early-type galaxy templates of various ages and metallicities. Although there are two free parameters-metallicity and age-for earlytype galaxy templates, we only use age as a meaningful parameter from fitting since the choice of metallicity is very limited $(Z=0.004,0.008$, and 0.02). Again, while the age itself is a model-dependent parameter (thus, there exists an "unphysical" age which is larger than the age of universe), we see that the amount of MIR excess is mainly affected by the age of a galaxy.

\subsection{The Nature of Intermediate MIR-Excess Galaxies}

In Section 3.1 (Figure 2), we show that there are a large number of galaxies that show MIR excess while lying on the tight optical red sequence at the same time. They fall on to the "intermediate" $(-1.2<N 3-S 11<0.2)$ or "weak" 


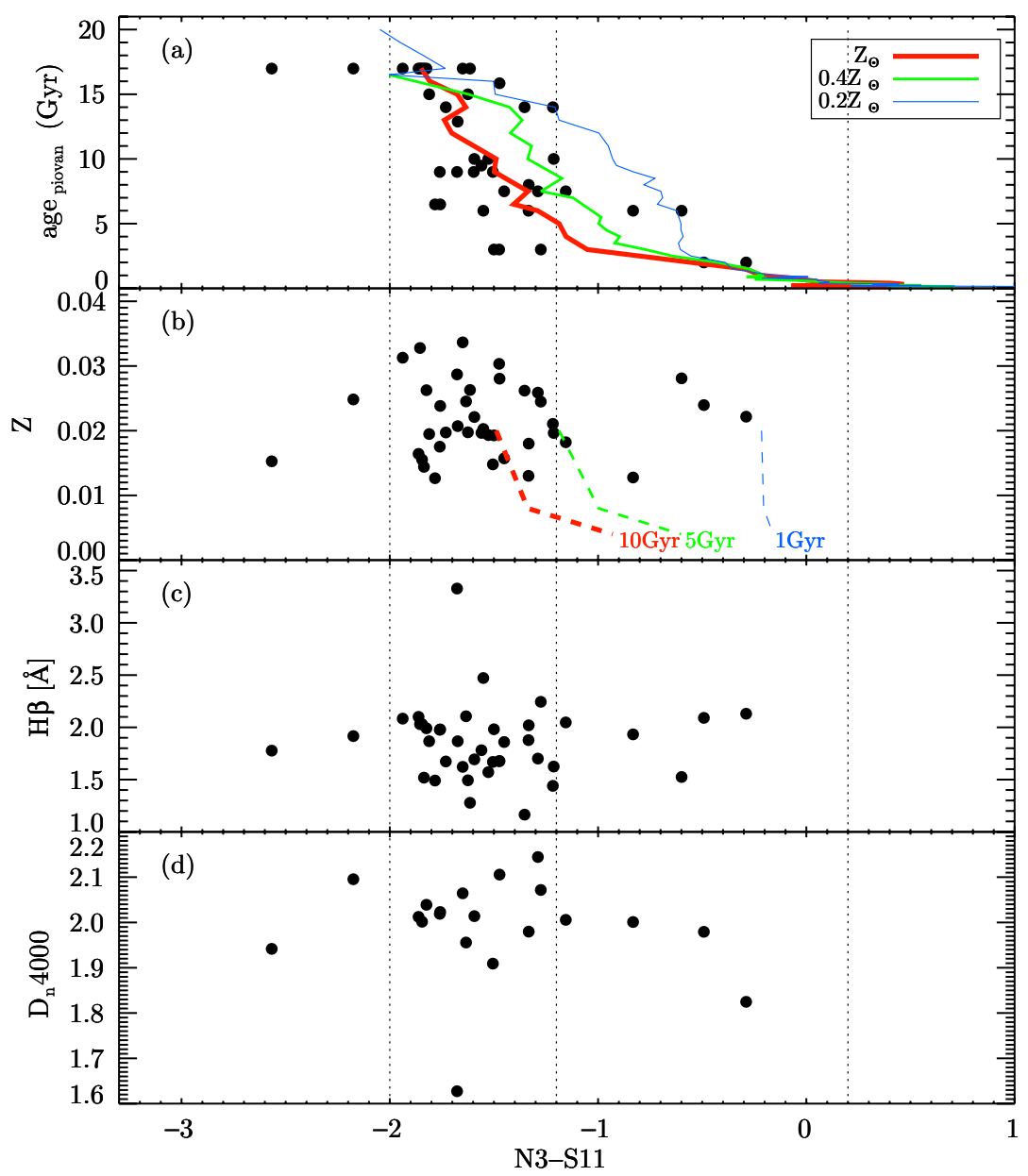

Figure 6. Relation between MIR excess ( $x$-axis, $N 3-S 11$ ) and (a) age derived from SED fitting, (b) metallicity measured in the SDSS spectra (Gallazzi et al. 2005), (c) $\mathrm{H} \beta$ line indices measured using SDSS spectra (SDSS-MPA catalog; Section 2.4), and (d) $\mathrm{D}_{n}(4000)$ measured using SDSS spectra (Kauffmann et al. 2003). Filled circles represent member galaxies with metallicity, the $\mathrm{H} \beta$, and/or $\mathrm{D}_{n}(4000)$ measurements. The number of objects with metallicity and $\mathrm{H} \beta$ is 40 (panels (a), (b), and (c)), while there are 20 galaxies with $\mathrm{D}_{n}(4000)$ measurements in addition (panel (d)). Strong MIR-excess $(N 3-S 11>0.2)$ galaxies are not included in these plots since their MIR-emission mechanism is different from that of weak/intermediate MIR-excess galaxies. The dotted vertical lines indicate the criteria for dividing weak/intermediate/strong MIR-excess, i.e., $N 3-S 11=-2.0,-1.2$, and 0.2 .

(A color version of this figure is available in the online journal.)

$(-2.0<N 3-S 11<-1.2)$ MIR-excess categories (Figure 3). To check the origin of the weak/intermediate MIR excess, and provide a physical meaning of the intermediate MIR-excess galaxies, we investigate various properties of this population.

First, we checked the optical spectra of the intermediate MIRexcess galaxies with $r$-band magnitude brighter than 17.77 mag. We find no sign of emission lines, which indicates that the MIR excess of most of these galaxies is not due to the star formation activity (at least within the fiber aperture).

We also examined the relation between MIR excess and other spectral properties of red-sequence galaxies (including weak MIR-excess galaxies). The top panel of Figure 6 shows the $N 3-S 11$ colors versus the luminosity-weighted mean stellar ages derived from the SED fitting (see Section 3.3). The second panel from the top illustrates the $N 3-S 11$ colors versus the metallicities measured from the SDSS spectra (Gallazzi et al. 2005), next is the $N 3-S 11$ colors versus the $\mathrm{H} \beta$ absorption line equivalent widths (Gallazzi et al. 2005), and the final panel shows the $N 3-S 11$ colors versus $\mathrm{D}_{n} 4000$ (Kauffmann et al. 2003). The SED-fitted ages are derived by fixing metallicity to the solar value, which should be a good approximation judging from Figure 6(b). Due to the limitation in modeling, there are "unphysical" ages that are larger than the age of the universe
( $\sim 17$ Gyr). The absolute age of the SEDs should not be taken too seriously, as these models are not meant to provide absolute ages (Piovan et al. 2003). These model fit parameters are meant to provide the relative age scales represented by different SED shapes. We find that the ages of the intermediate/weak MIR-excess galaxies derived by the SED fitting correlate with the $N 3-S 11$ colors (Figure 6(a)), with Spearman's rank correlation coefficient of -0.71 . Considering the degree of freedom at $38(\mathrm{~N}-2$, the number of points used is 40$)$, the correlation is reliable by more than $99.9 \%$ (Zar 1972). The correlation is already expected by the model color tracks overplotted in Figure 6(a). On the other hand, there is little correlation between metallicity and the $N 3-S 11$ color (Figure $6(\mathrm{~b}) ; r_{s}=0.008$, consistent with null hypothesis). Since the $N 3-S 11$ colors are more sensitive to age than metallicity, the MIR excess can be used to break the old age-metallicity degeneracies (e.g., Ko et al. 2009). Figures 6(c) and (d) show the relation between MIR excess and other well-known age indicators, the equivalent widths of Balmer absorption line $(\mathrm{H} \beta)$ and $\mathrm{D}_{n}(4000)$, a measure of the strength of the $4000 \AA$ break (Kauffmann et al. 2003). Spearman's rank correlation coefficient in this case is $r_{s}=-0.07$ and -0.14 , i.e., significance level (of the rejection of null hypothesis) greater than 50\% (degree of freedom at 38) 

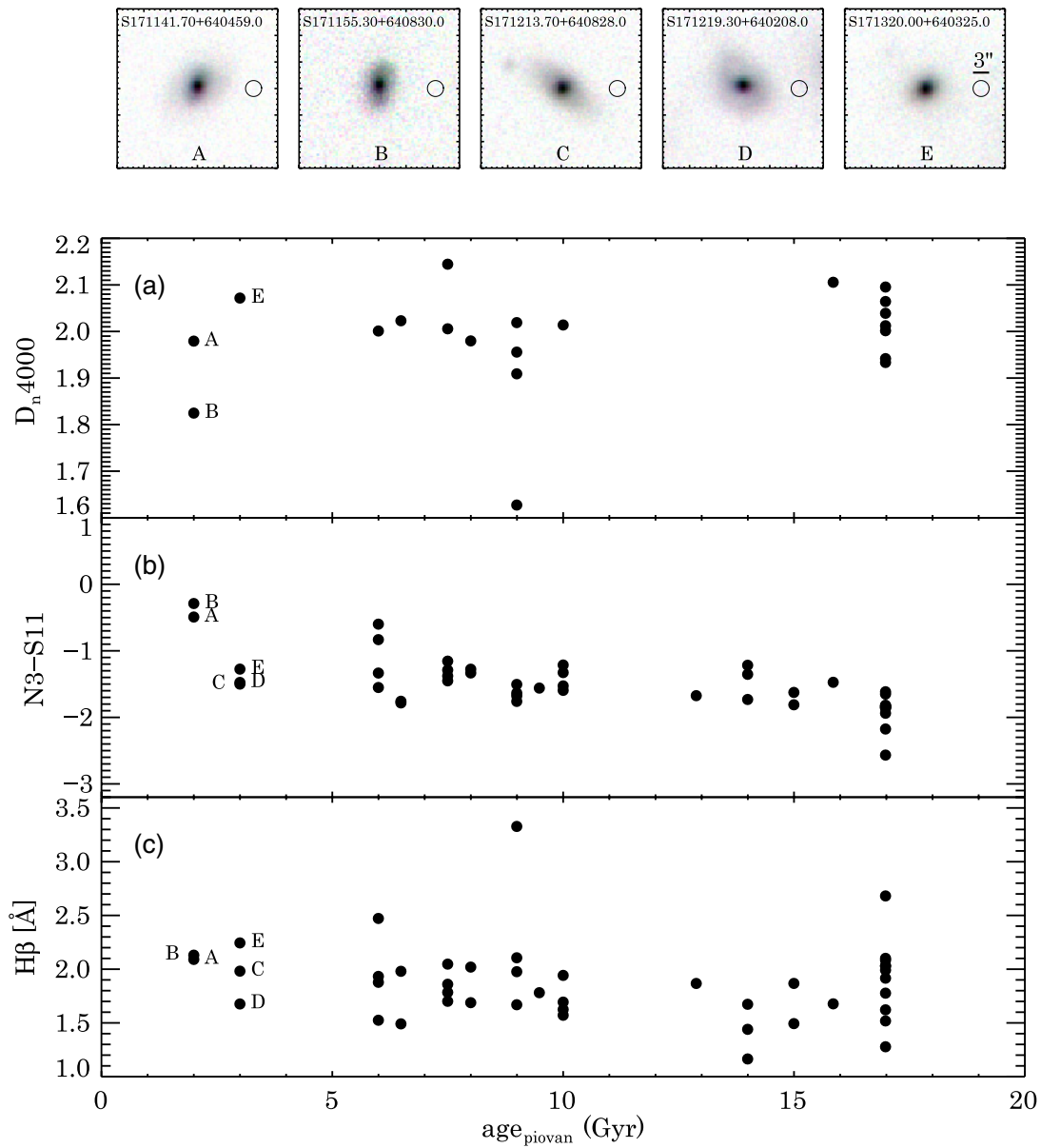

Figure 7. Relation between age derived from SED fitting ( $x$-axis) and (a) $\mathrm{D}_{n}(4000)$, (b) $N 3-S 11$, and (c) $\mathrm{H} \beta$ indices of intermediate/weak MIR-excess galaxies. The points plotted are the same as those in Figure 6. The different stellar age indicators $\left(\mathrm{D}_{n} 4000, N 3-S 11\right.$, and $\mathrm{H} \beta$ ) correlate with the stellar age but with large scatters. The SDSS $r$-band images of the youngest ( $<5 \mathrm{Gyr}$ ) galaxies are illustrated above to show their morphologies. The small circle in each image represents the SDSS fiber size, $3^{\prime \prime}$.

(A color version of this figure is available in the online journal.)

and $40 \%$ (degree of freedom at 18 ), respectively. Thus, the correlation between the MIR color and $\mathrm{H} \beta, \mathrm{D}_{n}(4000)$ is relatively weak.

Figure 7 illustrates the discrepancy between the different age indicators more clearly. The correlation coefficients between the SED-fitted age and age indicators are $r_{s}=0.14,-0.70$, and -0.18 for $\mathrm{D}_{n}(4000), N 3-S 11$, and the $\mathrm{H} \beta$ equivalent width, respectively. These represent significance levels smaller than $40 \%$, larger than $99 \%$, and smaller than $70 \%$, respectively. Only $N 3-S 11$ shows reliable correlation with the stellar age derived through SED fitting. This implies that the estimation of mean stellar age is not an easy task. Each age indicator has its pros and cons: $\mathrm{H} \beta$ is known to be less sensitive to metallicity compared to a simple color index such as $B-V$ as viewed from the stellar population synthesis modeling, yet it is also easily affected by emission line component. $\mathrm{D}_{n}(4000)$ is known to be a good tracer for young stellar age $\left(<1 \mathrm{Gyr}, \mathrm{D}_{n}(4000)<1.5\right.$; Balogh et al. 1999), yet highly affected by metallicity for older age (Kauffmann et al. 2003). Moreover, both $\mathrm{H} \beta$ and $\mathrm{D}_{n}(4000)$ is limited by the finite fiber size used for taking the SDSS spectra ( $3^{\prime \prime}$ diameter) that only samples the central part of a galaxy if it is extended. We show morphologies of five galaxies with the mean stellar ages from the SED fitting younger than $5 \mathrm{Gyr}$ (Figure 7). Most of the galaxies have outer disks and late-type morphology, and finite SDSS fiber size sample only the central regions indicated as a $3^{\prime \prime}$ bar in Figure 7 . Therefore, we address that MIR-excess is a relatively good age indicator that is free of finite spectroscopic aperture and age-metallicity degeneracy.

As the morphologies of five young galaxies $(<5 \mathrm{Gyr})$ suggest (Figure 7), the fraction of late-type morphologies is large for "intermediate" MIR-excess galaxies $(-1.2<N 3-S 11<0.2)$. We examine the morphologies of A2255 member galaxies at the bright end ( $r<16.5$ mag; Figure 5), since the visual morphological classification becomes difficult at the fainter magnitudes. While seven out of seven strong MIR-excess galaxies display late-type galaxy morphology, only five out of 25 (20\%) weak MIR-excess galaxies show late-type morphology which can easily be understood as a consequence of the well-known correlation between color and morphology of galaxies. In the case of the intermediate MIR-excess galaxies (S171251.20+640423.0 and S171225.70+641946.0 in Figure 5 and galaxies in Figure 7), they show late-type morphology such as disks despite being at the optical red sequence. The natural explanation of the colors and the morphologies of the intermediate MIR-excess galaxies is that these galaxies are late-type galaxies with small amount of star formation, very possibly in the process of quenching star formation. Studying E+A galaxies (i.e., characterized by old stellar population and strong Balmer absorption) would be 
another way to see the truncation of star formation, yet we did not find any $\mathrm{E}+\mathrm{A}$ galaxies using a criteria of $\mathrm{EW}(\mathrm{H} \delta)>5 \AA$ (Goto 2007, in addition to the criteria for no detectable [O II] and $\mathrm{H} \alpha$ emission lines) among the member galaxies with spectroscopic information. The average $\mathrm{EW}(\mathrm{H} \delta)$ of the intermediate MIR-excess galaxies is $\sim 1 \AA$, with a typical measurement uncertainty of $0.5 \AA$. Only $\sim 50 \%$ of the intermediate MIR excess galaxies are spectroscopically observed, thus it is not clear whether the remaining $\sim 50 \%$ would be classified as E+A galaxies or not. This result demonstrates an advantage of using MIR excess in studying the transition population over optical spectra. The "weakened" star formation activity in intermediate MIRexcess galaxies, regardless of its origin (weak star formation or the AGB-dust), is naturally explained by the idea that these galaxies are in the process of transformation from star-forming galaxies (strong MIR-excess galaxies) to quiescent galaxies (weak MIR-excess galaxies) at optical red sequence. The relation with these transformation and the cluster environment will be described in more detail in Section 5.3.

\subsection{Contribution of AGNs to MIR-excess Galaxies Sample}

AGNs cause MIR excess in galaxies (Quillen et al. 1999) as well as ongoing star formation, and thus alter the derived SFRs of galaxies. The different diagnostics for AGNs, such as infrared color and optical line ratio, produce a significantly different result for demarcating AGNs and star-forming galaxies among MIR-excess galaxies (Brand et al. 2009). Therefore, we do not force the differentiation of AGNs from our MIRexcess galaxies in this paper. Instead, we briefly discuss how many AGNs are included in MIR-excess galaxies and how they affect our analysis and conclusion, using the previously known AGNs detected in either X-ray or radio, classified as AGNs using optical line diagnostics (Miller \& Owen 2003; Davis et al. 2003).

In the AKARI/Spitzer FOV, there lie 13 previously known AGNs. Among the 13 AGNs, 10 are detected in either $11 \mu \mathrm{m}$ (S11) or $24 \mu \mathrm{m}$ (L24/MIPS $24 \mu \mathrm{m}$ ) images, and 3 are not. Ten AGNs detected in $11 \mu \mathrm{m}$ or $24 \mu \mathrm{m}$ are radio sources with $1.4 \mathrm{GHz}$ flux larger than $0.35 \mathrm{mJy}$, while three are Seyferts and seven are objects with old stellar population either with weak [N $\mathrm{NI}_{\mathrm{II}}$ or $\left[\mathrm{S}_{\mathrm{II}}\right]$ emission lines, or without any signs of emission lines (Miller \& Owen 2003). By number, AGNs contribute $3 \%$ of the star-forming galaxies, and $15 \%$ of the weak MIR-excess galaxies. No AGN cross-identification is found in intermediate MIR-excess galaxies. The contribution from three Seyfert galaxies to the total SFR is less than $5 M_{\odot} \mathrm{yr}^{-1}$, i.e., less than $4 \%$ of $\sum_{>0.25 M_{\odot} \mathrm{yr}^{-1}}$ SFR (see Section 4.2 for details). With this little contribution, we conclude that our main analysis and conclusion is not affected by the presence of AGNs among MIRexcess galaxies especially in the sense that we mainly discuss star-forming galaxies and intermediate MIR-excess galaxies in A2255.

\section{STAR FORMATION RATES}

As we have shown in the previous section, the strong MIRexcess galaxies $(N 3-S 11>0.2)$ are galaxies with ongoing star formation. In this section, we discuss how much star formation is hidden in IR (e.g., Bai et al. 2007), and compare the total SFR of A2255 with those of other galaxy clusters to see if there is anything special in the star formation activity of A2255. Only galaxies with strong MIR-excess are considered here for the SFR study, since SFRs in other classes of MIR-excess galaxies are minimal, less than $0.1 M_{\odot} \mathrm{yr}^{-1}$.

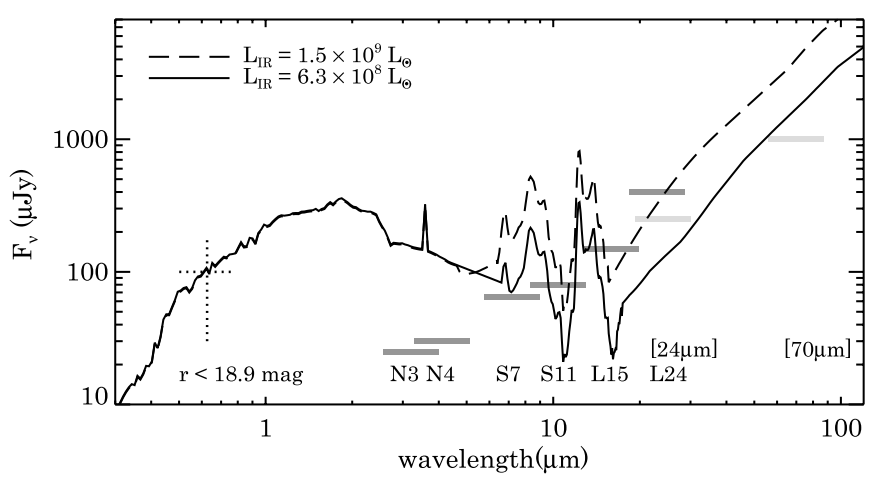

Figure 8. Comparison between star-forming galaxy templates with different IR luminosities and the flux limits in each filter. The overplotted lines are star-forming galaxy templates with $L_{\mathrm{IR}}=6.3 \times 10^{8} L_{\odot}$ (dashed line) and $L_{\mathrm{IR}}=1.5 \times 10^{9} L_{\odot}$ (solid line). The limits (shaded region) indicate $5 \sigma$ flux limits over $2 \times$ FWHM diameter in all filter bands (see Table 1 ). When optical magnitude limit for complete redshift identification, $r<18.9 \mathrm{mag}$ (see Section 2.2), is applied, the limits in the derived IR luminosity varies between $L_{\mathrm{IR}}>(0.6-1.5) \times 10^{9} L_{\odot}$; on average, the minimum SFR we derive through SED fitting is $>0.1 M_{\odot} \mathrm{yr}^{-1}$ according to the IR luminosity limit of $L_{\text {IR }}>(0.6-1.5) \times 10^{9} L_{\odot}$. To be conservative, we treat our MIR data as complete above $L_{\mathrm{IR}}>1.5 \times 10^{9} L_{\odot}$.

\subsection{Infrared Star Formation Rate}

We calculate SFRs of member galaxies from the derived IR luminosity through the SED fitting (Section 3.3, Figure 4), and compare the SFR with those from other wavelengths. The total IR luminosities of A2255 member galaxies range from $1.0 \times 10^{8}$ to $3.2 \times 10^{10} L_{\odot}$, while the median value is $\left\langle L_{\mathrm{IR}}\right\rangle \simeq 2.8 \times 10^{9} L_{\odot}$. These values correspond to $\sim 0.1 M_{\odot} \mathrm{yr}^{-1}<\mathrm{SFR}(\mathrm{IR}) \lesssim 7.0 \mathrm{M}_{\odot} \mathrm{yr}^{-1}$ when converted using the Kennicutt (1998) relation between $L_{\mathrm{IR}}$ and SFR. However, considering the flux limits in MIR and cluster member completeness limit in optical, the conservative limit in $L_{\mathrm{IR}}$ in our survey is $L_{\mathrm{IR}}>1.5 \times 10^{9} L_{\odot}$ (Figure 8$)$. We do not find any luminous IR galaxy $\left(L_{\mathrm{IR}}>10^{11} L_{\odot}\right)$ candidates in A2255.

We compare the "IR-derived SFR" with SFRs derived using other star formation indicators (Figure 9) at different wavelengths. Figure 9(a) shows the comparison between SFR $_{\mathrm{IR}}$ and $\mathrm{SFR}_{\mathrm{H} \alpha}$ (cor.), i.e., IR-derived SFR and the $\mathrm{H} \alpha$-derived SFR corrected for dust extinction ("cor." means the luminosity is corrected for internal dust extinction). In order to derive $\mathrm{SFR}_{\mathrm{H} \alpha}$ (cor.), we first derive $\mathrm{SFR}_{\mathrm{H} \alpha}$ (uncor.), SFR based on $\mathrm{H} \alpha$ flux not corrected for the internal dust extinction, using $\mathrm{H} \alpha$ fluxes from the SDSS-MPA catalog (see Section 2.4) and the $\mathrm{H} \alpha$ SFR conversion formula of Kennicutt (1998). Since the SDSS spectra are obtained using fibers with a fixed size of $3^{\prime \prime}$ diameter, we adopt the aperture correction for each object from Brinchmann et al. (2004), i.e., $f_{\text {total }} / f_{\text {fiber }}{ }^{13}$ The "extinction" in $\mathrm{H} \alpha$ flux $-A(\mathrm{H} \alpha)$-is estimated from the Balmer decrement $(\mathrm{H} \alpha / \mathrm{H} \beta$ flux ratio), by adopting a case $\mathrm{B}$ recombination at $T=10,000 \mathrm{~K}$ (Osterbrock 1989) and the assumption of the Calzetti et al. (2000) extinction law for starburst galaxies. The median amount of extinction in $\mathrm{H} \alpha$ is $\langle A(\mathrm{H} \alpha)\rangle=0.78 \mathrm{mag}$ (Figure 9(b)). There is a weak correlation between SFRs and $A(\mathrm{H} \alpha)$, i.e., galaxies with larger SFRs having larger $A(\mathrm{H} \alpha)$.

We also compare the UV-derived SFR with the IR-derived SFR (Figure 9(c)), using the GALEX NUV flux as a measure

\footnotetext{
13 Note that Brinchmann et al. (2004) also provide the spectroscopic SFRs by spectral line fitting; however, we find that their SFRs significantly depend on the optical colors of galaxies, reflecting the past star formation history of galaxies. Since we focus on the current ongoing SFR in A2255 members, we just use the $\mathrm{H} \alpha$ line to derive the optical SFR.
} 

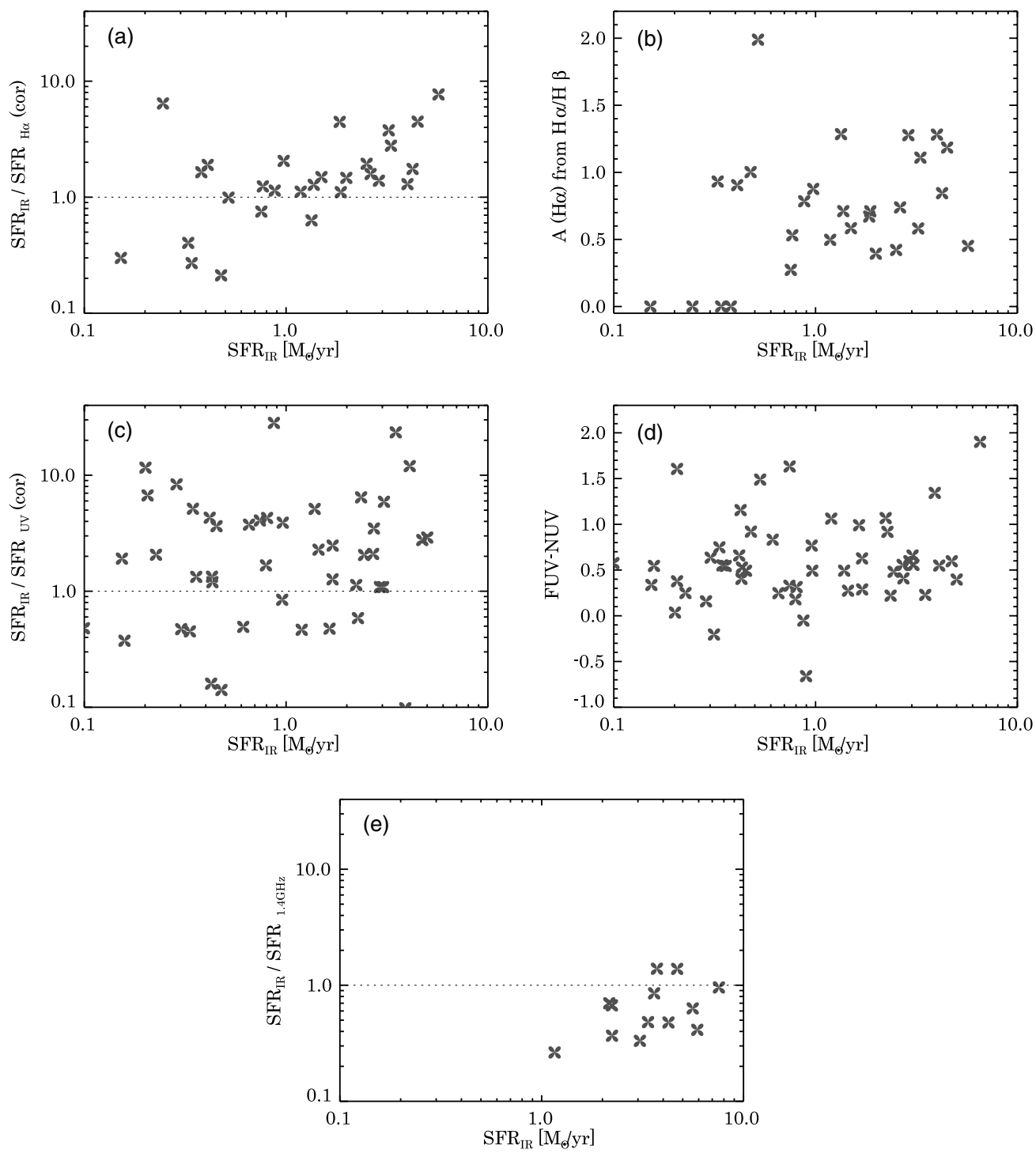

Figure 9. Ratio between $\mathrm{SFR}_{\mathrm{IR}}$ and SFR from different indicators ((a) SFR $\mathrm{IR}_{\mathrm{IR}} / \mathrm{SFR}_{H \alpha}$, (c) $\mathrm{SFR}_{\mathrm{IR}} / \mathrm{SFR}_{\mathrm{UV}}$, (e) $\mathrm{SFR} \mathrm{IR}_{\mathrm{IR}} / \mathrm{SFR}_{1.4 \mathrm{GHz}}$ ) in addition to the dust attenuation measures in $\mathrm{H} \alpha$ (b) and $\mathrm{UV}$ (d). In panels (a) and (b), $\mathrm{SFR}_{\mathrm{H} \alpha}$ and $\mathrm{SFR}_{\mathrm{UV}}$ are extinction corrected using Balmer decrement and $\mathrm{UV}$ slope $\beta$, respectively. SFR $\mathrm{H} \alpha$ is derived using the $\mathrm{H} \alpha$ line flux measured in SDSS optical spectra. The mean extinction from the Balmer line ratio is $\langle\mathrm{A}(\mathrm{H} \alpha)\rangle \sim 0.78$ mag, suggesting a factor of $\sim 2$ extinction in $\mathrm{SFR}_{\mathrm{H} \alpha}$. The (FUV - NUV) color is another measure of dust extinction that reflects UV slope $\beta$.

of $\mathrm{SFR}_{\mathrm{UV}}$. As in the case of $\mathrm{H} \alpha$, we derive the extinction correction in UV wavelengths from the UV slope $\beta$ inferred by FUV - NUV colors (Meurer et al. 1999). Star-forming galaxies in A2255 show relatively blue FUV - NUV colors of $\langle$ FUV $-\mathrm{NUV}\rangle=0.55 \mathrm{mag}$ (Figure 9(d)). The mean extinction correction derived is $\langle A(\mathrm{UV})\rangle \sim 1.2 \mathrm{mag}$. When this correction is applied (SFR IR $_{\text {versus }} \mathrm{SFR}_{\mathrm{UV}}$ (cor); Figure 9(c)), the two SFRs are comparable with each other. Both the $\mathrm{H} \alpha$ - and the UVderived SFRs agree with the IR-derived SFR when corrected properly for the internal dust extinction, albeit with a large scatter $(>\times 2)$.

Finally, in Figure 9(e), we present the comparison between $\mathrm{SFR}_{\mathrm{IR}}$ and $\mathrm{SFR}_{\text {radio }}$ using $1.4 \mathrm{GHz}$ flux in Miller \& Owen (2003). The conversion formula from $1.4 \mathrm{GHz}$ flux to $\mathrm{SFR}_{\text {radio }}$ is adopted from Bell (2003). Although the number of the matched member galaxies is small, SFRs from the two indicators differ by about a factor of two, with the SFR $\mathrm{IR}_{\mathrm{R}}$ being systematically larger than $\mathrm{SFR}_{\text {radio }}$.

Overall, we find a conventional extinction correction to the dust extinction works roughly well for estimating SFRs in individual galaxies (e.g., Choi et al. 2006), but such correlations accompany large scatters. Therefore, we consider the usage of the IR data is a robust way to derive the star formation compared to UV and the optical data.

\subsection{Total Star Formation Rate of A2255}

In this section, we derive the global properties of star formation activity in A2255, in order to examine if the merging activity enhanced the star formation activity of A2255 in particular. This is done by deriving the "total" SFR in A2255 by constructing the IR luminosity function and comparing the total SFR of A2255 with other galaxy clusters. The construction of the luminosity function is done using the following equation:

$$
\phi\left(\log L_{i}\right)=\frac{1}{A} \frac{n}{\Delta(\log L)},
$$

where $A$ is the surveyed area, $n$ is the number of galaxies whose luminosity falls within the $i$ th bin, and $\Delta(\log L)$ is the luminosity bin size. We used the survey coverage of $\sim 2000 \mathrm{arcmin}^{2}$, i.e., $\sim 16 \mathrm{Mpc}^{2}$ in physical scale. 

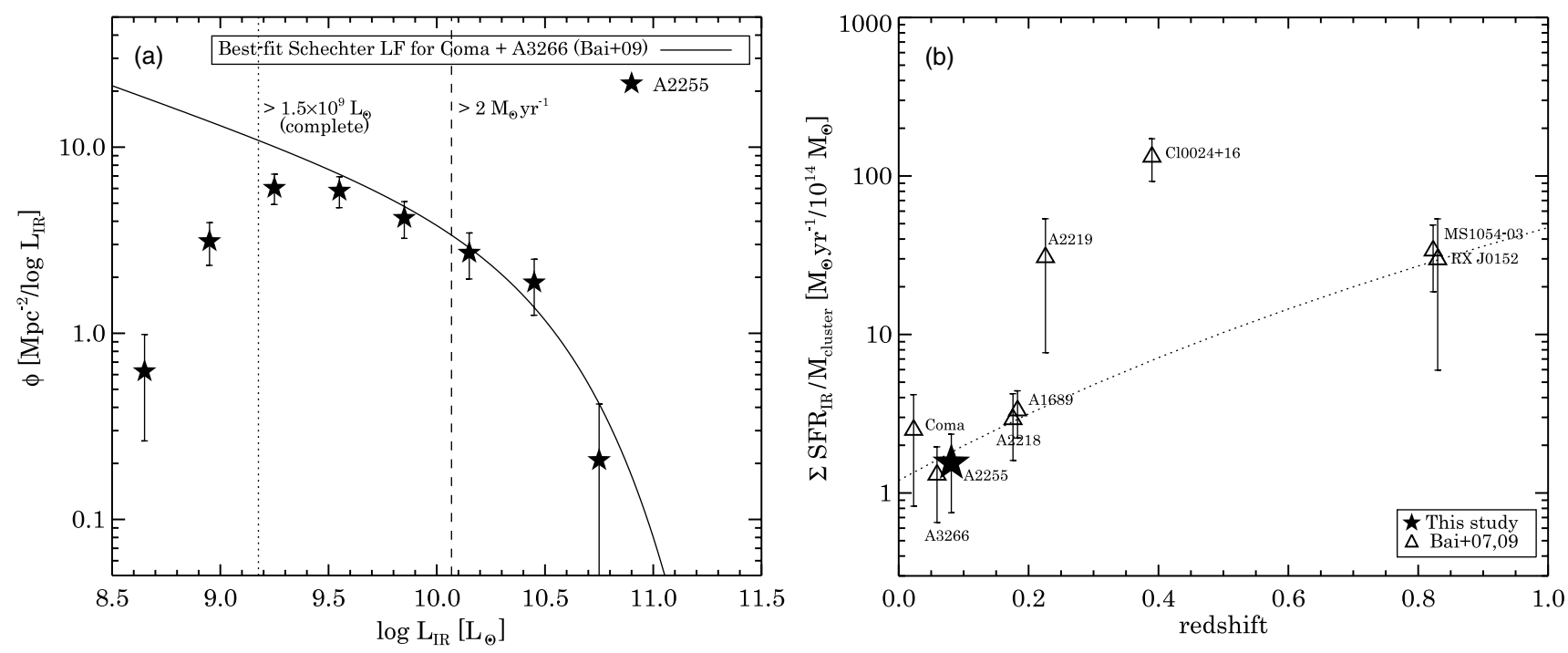

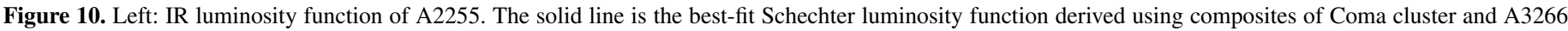

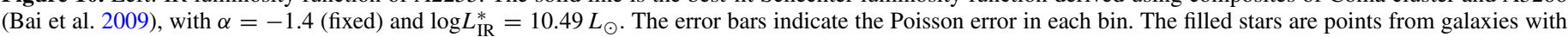

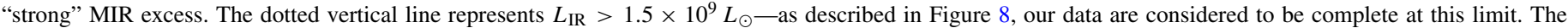

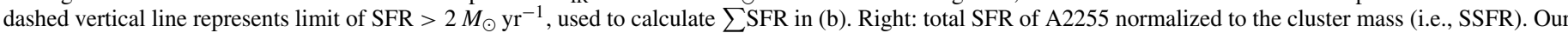

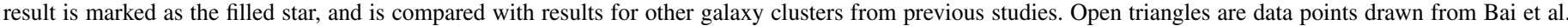
(2007, 2009). The dotted line represents $(1+z)^{5.3}$, an evolutionary trend of SSFR for galaxy clusters (Bai et al. 2009).

We present the derived IR luminosity function with the Poisson error bars in Figure 10(a). The overplotted solid line is the best-fit Schechter luminosity function for Coma and A3266 composite (Bai et al. 2009), with parameters $\alpha=-1.41$ and $\log L_{\mathrm{IR}}^{*}\left(L_{\odot}\right)=10.49$. The IR luminosity function of A2255 is consistent with those of Coma and A3266 in terms of both the shape and the normalization above the completeness limit of IR luminosity $\left(L_{\mathrm{IR}} \sim 1.5 \times 10^{9} L_{\odot}\right.$; Figure 8$)$.

Comparing the IR luminosity functions of Coma and A3266, Bai et al. (2009) suggested a possibility that local galaxy clusters share a universal IR luminosity function. A2255 is close to the Coma cluster in terms of cluster mass $\left(\sim 10^{15} M_{\odot}\right.$; Burns et al. 1995; Lokas \& Mamon 2003), but has a slightly lower mass than A3266 ( $3.3 \times 10^{15} M_{\odot}$; Bai et al. 2009). In terms of the dynamical status, A2255, A3266, and the Coma cluster all share similar properties - these galaxy clusters are suggested to be in the late phase of the cluster-cluster merger (e.g., Watanabe et al. 1999; Sauvageot et al. 2005). The consistency of the A2255 IR luminosity function with those of Coma and A3266 supports the idea of universal IR luminosity function in local galaxy clusters (e.g., Bai et al. 2009), at least when they are in the late stage of cluster-scale merger. Yet it should be noted that the number of galaxy clusters studied is small, and most galaxy clusters studied to date are biased to a dynamically unrelaxed system.

With the derived IR luminosity function, we calculate the "total" SFR in A2255 by integrating the IR luminosity function. In the luminosity range of $L_{\mathrm{IR}}>1.5 \times 10^{9} L_{\odot}$, i.e., $\mathrm{SFR}(\mathrm{IR})>$ $0.25 M_{\odot} \mathrm{yr}^{-1}$, the integration of the IR luminosity function yields $\sum_{>0.25 M_{\odot} \mathrm{yr}^{-1}}^{\mathrm{LF}} \mathrm{SFR}=115 M_{\odot} \mathrm{yr}^{-1}$ for $\mathrm{A} 2255$. This is consistent with the summation of SFRs of "individual" member galaxies, $\sum_{>0.25 M_{\odot} \mathrm{yr}^{-1}} \mathrm{SFR}=131 M_{\odot} \mathrm{yr}^{-1}$.

In the SFR summation of individual star-forming galaxies $\left(\sum_{>0.25 M_{\odot} \mathrm{yr}^{-1}} \mathrm{SFR}=131 M_{\odot} \mathrm{yr}^{-1}\right)$, we split the contribution to the SFR from spectroscopic member galaxies and photometric member galaxies in order to see the effects from the cluster membership determination. Among $131 M_{\odot} \mathrm{yr}^{-1}$, the member galaxies with spectroscopic redshifts contribute $103 M_{\odot} \mathrm{yr}^{-1}$, and the member galaxies with photometric redshifts contribute
$28 M_{\odot} \mathrm{yr}^{-1}$. The contribution from galaxies with photometric redshifts (i.e., optically faint member galaxies) is about $20 \%$ : therefore, even at the very unlikely case that all photometric members turn out to be non-members, it does not have a strong effect on the derived total SFR.

In order to compare the star formation activity of A2255 with the other galaxy clusters, we use a "specific SFR" (SSFR) of the galaxy cluster-the total SFR normalized by the cluster virial mass $\left(\sum \mathrm{SFR} / \mathrm{M}_{\mathrm{cl}}\right)$. The result is marked as a filled star in Figure 10(b). The $\sum \mathrm{SFR} / \mathrm{M}_{\mathrm{cl}}$ value is calculated by integrating SFRs of galaxies with SFRs larger than $2 M_{\odot} \mathrm{yr}^{-1}$ and within a $0.5 r_{200}$ radius, in order to facilitate the comparison with the mass-normalized SFR of other clusters (other points; Bai et al. 2007, 2009). The points for Coma, A3266, MS105403, and RX J0152 (open triangles in Figure 10(b)) are from the Spitzer MIPS $24 \mu \mathrm{m}$ studies (Bai et al. 2007, 2009), while the points for A2218, A1689, A2219, and C10024+16 are from ISO observation (Bai et al. 2007). The sum of the A2255 SFR is $\sum \operatorname{SFR}_{\mathrm{IR}}\left(>2 \mathrm{M}_{\odot} \mathrm{yr}^{-1}\right.$, within $\left.0.5 \mathrm{r}_{200}\right)=15.5 \mathrm{M}_{\odot} \mathrm{yr}^{-1}$. We use $r_{200}=2.10 \mathrm{Mpc}$ and $M_{\mathrm{cl}}=0.45-1.3 \times 10^{15} M_{\odot}$ from previous studies (Neumann 2005; Burns et al. 1995; Feretti et al. 1997). The derived SSFR of A2255 is comparable to those of four other clusters at $z<0.2$. On the other hand, compared to two intermediate-redshift clusters, A2219 and C10024+16, the SSFR of A2255 is more than an order of magnitude lower. From the study of the total SFR in A2255, we find no evidence that the overall star formation activity is enhanced in A2255 compared to other clusters at low to intermediate redshifts.

\section{ENVIRONMENTAL DEPENDENCE OF STAR FORMATION AND GALAXY TRANSFORMATION}

We investigate the correlation between the galaxy evolution and their environment to see the role of the environment in the evolution of A2255 member galaxies. First, we define the parameters that represent the local environment of galaxies. Second, we examine how different levels of MIR excess correlate with the environment. 


\subsection{Substructures and Kinematic Characteristics in A2255}

We use the local surface density of galaxies and clustercentric radius as indicators of environment. The local surface density of galaxies is expressed as $\Sigma_{5 \text { th }}$, the density of member galaxies (with either spectroscopic or photometric redshifts) within a circle whose radius is the distance to the fifth nearest galaxy in comoving scale. Galaxies brighter than $r \sim 19$ mag are used for calculating the local density. If a galaxy cluster is simply a relaxed system, the local surface density would monotonically decrease as the clustercentric distance increases. However, for clusters like A2255, this is not the case. We identify substructures with densities higher than the local average densities to be areas that deviates from a smooth projected number density represented by the Navarro-Frenk-White (NFW) function (Navarro et al. 1997). To fit the relation between the clustercentric distance versus the observed $\Sigma_{5 \text { th }}$, we use a twodimensional projected form of the NFW function (Elíasdóttir \& Möller 2007; see the solid line in Figure 11(a)):

$$
\begin{aligned}
& \Sigma_{\mathrm{nfw}}=\frac{2 r_{s} \delta_{c} \rho_{c}}{1-X^{2}}\left(1-\frac{2}{\sqrt{1-X^{2}}} \operatorname{arctanh} \sqrt{\frac{1-X}{1+X}}\right), X<1 \\
& \Sigma_{\mathrm{nfw}}=\frac{2 r_{s} \delta_{c} \rho_{c}}{X^{2}-1}\left(1-\frac{2}{\sqrt{X^{2}-1}} \arctan \sqrt{\frac{X-1}{1+X}}\right), X>1
\end{aligned}
$$

In this equation, $X$ indicates $R_{\mathrm{cl}} / r_{s}$, where $R_{\mathrm{cl}}$ is the clustercentric radius. The parameters $r_{s}, \delta_{c}$, and $\rho_{c}$ represent the scale radius, the characteristic overdensity, and the critical density. Our best-fitted parameters are $r_{s}=0.68 \mathrm{Mpc}$ and $\delta_{c} \rho_{c}=127 \mathrm{Mpc}^{-3}$. The galaxies above $\sigma_{\mathrm{rms}}$ from the fitted line are considered as galaxies at substructures with a locally high density of galaxies. The coordinates of these galaxies are plotted as open circles in Figure 11(b), over the distribution of all member galaxies in the Y03 catalog. The overplotted thick lines are contours of "galaxies at high density environment," and we define these peaks as substructures. This means that it is not necessary that all substructure galaxies have high $\Sigma_{5 \text { th }}$, although most of them do. The locations of these structures are consistent with galaxy number density contour. The coordinates of the substructures marked in Figure 11(b) are (17:13:44, 64:14:42) for substructure "V," (17:14:48, 64:10:00) for "W," (17:13:10, 64:05:00) for "X," (17:12:20, 64:00:00) for "Y," and (17:11:40, 64:08:00) for "Z." Substructures A, B, C, D, and E are consistent with the substructures defined in Yuan et al. (2003) with the same alphabets. They first defined the substructures from the peak of number density contour $\left(>0.15\right.$ galaxies $\operatorname{arcmin}^{-2}$; see Figure 5 of Yuan et al. 2003). By studying the velocity distribution of galaxies in the substructures, these authors confirm that substructures $\mathrm{A}, \mathrm{B}$, and $\mathrm{C}$ have different velocity components compared to the main cluster, while substructures D and E might be a result of a projection effect. Our MIR observation is limited to within the central $\sim 2 \mathrm{Mpc}$ radius of A2255. Consequently, the substructures are all defined within this $\sim 2 \mathrm{Mpc}$ radius circle (about virial radius of the cluster).

Besides the substructures, we also investigate the large-scale distribution of galaxies around A2255, since A2255 is known to be a member of the North Ecliptic Pole (NEP) supercluster (e.g., Mullis et al. 2001). Understanding the large-scale distribution of galaxies will be helpful to see how the distribution of galaxies in A2255 is connected to the features such as walls and filaments. Figure 12(a) shows the redshift distribution of galaxies located in a $3 \times 3 \mathrm{deg}^{2}$ region surrounding A2255. The spectroscopic
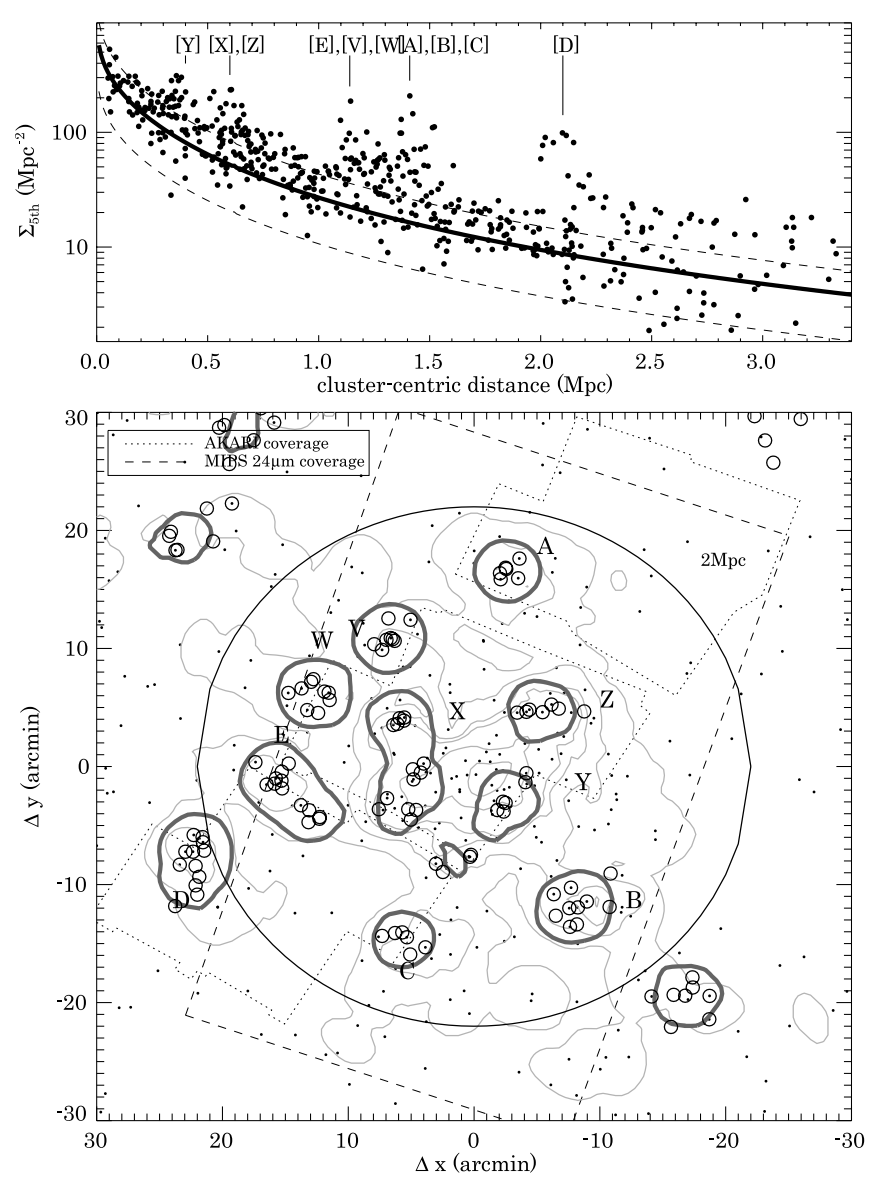

Figure 11. Top: local galaxy surface density as a function of clustercentric distance. Local galaxy surface density is expressed as $\Sigma_{5 \text { th }}$, galaxy number density within a circle with a radius of a distance to the fifth nearest galaxy. The overplotted thick solid line has a form of two-dimensional projection of the NFW profile for dark matter halos (Navarro et al. 1997; projection form adopted from Elíasdóttir \& Möller 2007). The best-fit line is derived by excluding the outliers iteratively. The dashed lines indicate $\pm \sigma_{\text {rms }}$ from the best-fit solid line. There are several peaks of galaxies with $\Delta\left|\Sigma_{5 \text { th }}\right|>\sigma_{\text {rms }}$, i.e., galaxies showing larger local surface density compared to the expected surface density from a relaxed system. These peaks of galaxies are defined as "substructure"s A-E, and $\mathrm{V}-\mathrm{Z}$ in the bottom panel. Bottom: spatial distribution of A2255 member galaxies. The overlaying contours are number density contours of galaxies with spectroscopic redshifts of $0.068<z<0.095$. Open circles indicate "galaxies at high-density region," which lies in the peaks of the top panel.

redshifts are obtained from SDSS DR7 (Abazajian et al. 2009). While the solid histogram shows the redshift distribution of all the galaxies within a circle with $10 \mathrm{Mpc}$ radius centered on A2255, the filled histogram indicates the redshift distribution of galaxies within $2 \mathrm{Mpc}$ distance from the cluster center. The large-scale analysis shows strong redshift peak at $z \sim 0.08$ which can be attributed to the presence of A2255 and the NEP supercluster. On the other hand, in the central region within the clustercentric distance of $2 \mathrm{Mpc}$, the redshift distribution is far different from a single Gaussian distribution: the possibility that the distribution of galaxies located within the central 2 Mpc follows a single Gaussian function is only $40 \%$ by the Kolmogorov-Smirnov test. Instead, the redshift distribution is well described by the sum of two redshift components with $z_{1}=0.077 \pm 0.0027$ and $z_{2}=0.082 \pm 0.0017$, with a lack of galaxies at $z \sim 0.08$. Figures 12(b)-(e) show the spatial distribution of galaxies at different redshift bins. The galaxies at the blue velocity component (b; $0.07<z<0.078$ ) and the red velocity component $(\mathrm{d} ; 0.081<z<0.084)$ are concentrated in the cluster center. The redshift range of 

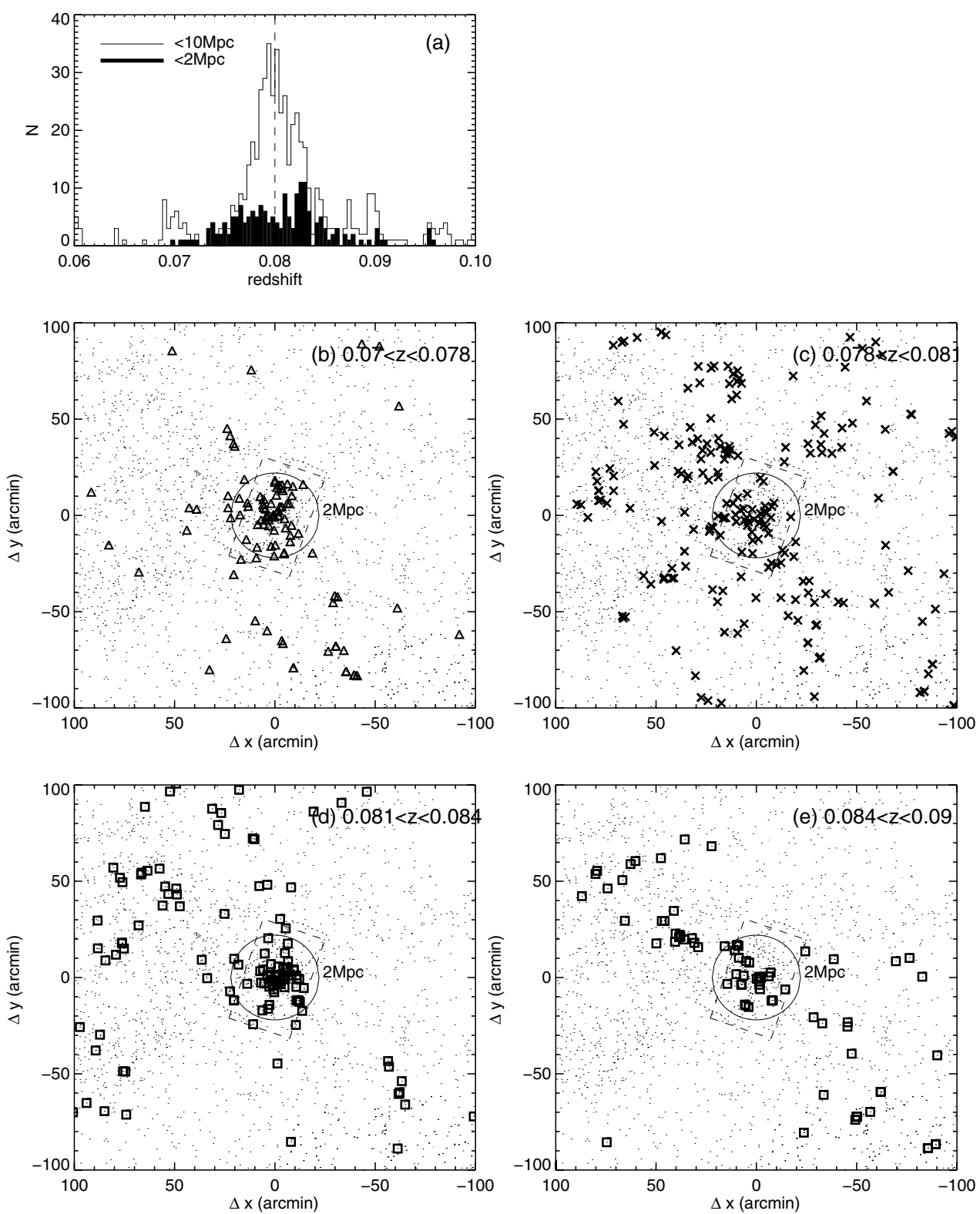

Figure 12. (a) Spectroscopic redshift distribution of A2255 member galaxies and galaxies in the vicinity of A2255. While galaxies within a $<10 \mathrm{Mpc}$ distance from the A2255 center show the redshift distribution peaked at $z \sim 0.08$ (dashed line), the redshift distribution of A2255 member galaxies is skewed to have two distinct velocity peaks $\left(z_{1}=0.077\right.$ and $\left.z_{2}=0.082\right)$. (b) Spatial distribution of galaxies at $0.07<z<0.078$. The circle indicates a $2 \mathrm{Mpc}$ radius circle, and the dotted/dashed line shows the fields of view of the AKARI IRC/Spitzer MIPS. (c) Spatial distribution of galaxies at $0.078<z<0.081$. (d) Spatial distribution of galaxies at $0.081<z<0.084$. (e) Spatial distribution of galaxies at redshifts $0.084<z<0.09$.

$0.078<z<0.081$, where only few of the A2255 cluster members reside in, consists of galaxies that are distributed like a flat sheet at $z \sim 0.08$ (Figure 12(c)). At the reddest velocity tail (e; $0.084<z<0.09$ ), galaxies are distributed along a filamentary structure that pass through A2255. The two velocity groups $(0.07<z<0.078$ and $0.081<z<0.084)$ are virtually superimposed in the sky. The velocity dispersion of two groups are $800 \mathrm{~km} \mathrm{~s}^{-1}$ (blue) and $500 \mathrm{~km} \mathrm{~s}^{-1}$ (red). Using simple virial theorem, the masses of each component are $1.7 \times 10^{15} M_{\odot}$ (blue) and $6.7 \times 10^{14} M_{\odot}$ (red) when virial radius is $2 \mathrm{Mpc}$ for each group. According to the criteria for gravitationally bounded two-body problem (Beers et al. 1982; Tran et al. 2005), the probability that these two components are gravitationally bounded is when the following equation is satisfied:

$$
V_{r}^{2} R_{p} \leqslant 2 G M \sin ^{2} \alpha \cos \alpha
$$

In this equation, $V_{r}$ is the relative velocity between the two groups ( $1500 \mathrm{~km} \mathrm{~s}^{-1}$ ), $R_{p}$ is the projected separation (less than $\sim 100 \mathrm{kpc}$ since two components are nearly superimposed), $M$ is the total mass of the system and $\alpha$ is the projected angle with respect to the plane of the sky. The equation is valid for the projection angle range of $6^{\circ}<\alpha<89^{\circ}$, and thus the probability that the two groups are bounded is more than $90 \%$. Therefore, A2255 is clearly thought to be a "merging" galaxy cluster with two different velocity peaks.

\subsection{Environmental Dependence of Star Formation}

Figures 13(a) and (b) show the projected two-dimensional spatial distribution of star-forming galaxies in A2255. The color of each point indicates the redshift, and the size of each point is proportional to the SSFR. The figures show that galaxies 

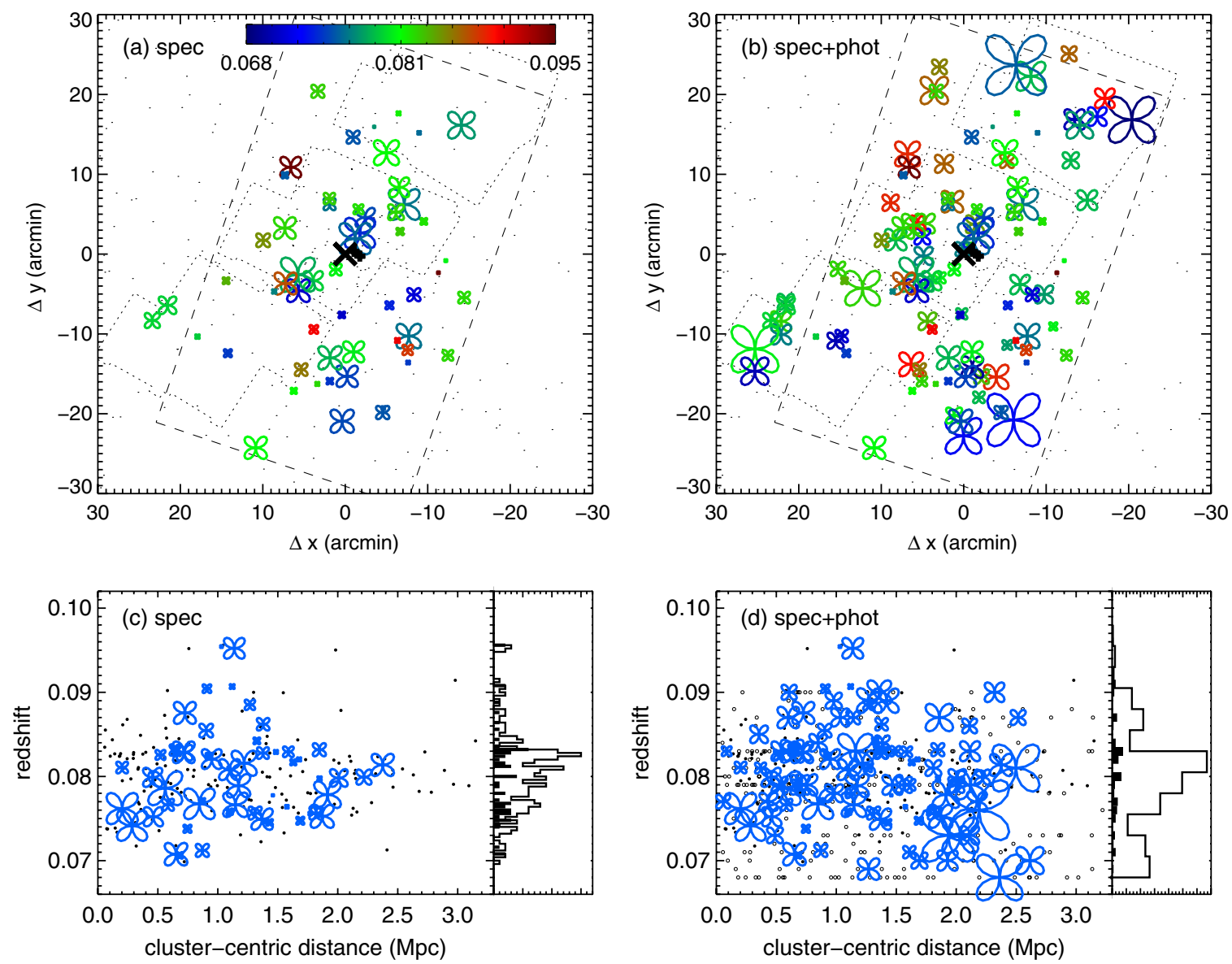

Figure 13. (a) Two-dimensional spatial distribution of star-forming galaxies in A2255. Galaxies with spectroscopic redshifts are plotted as clovers. The symbol color denotes the redshift of a galaxy as indicated in the color bar, and the symbol size is proportional to the SSFR. (b) Same as in panel (a); galaxies with either spectroscopic or photometric redshifts are plotted. (c) The clustercentric distance and velocity distribution of star-forming galaxies in A2255. The points plotted as small dots are all member galaxies with spectroscopic redshifts defined in the Y03 catalog. Overplotted clovers are star-forming galaxies, with symbol size proportional to the SSFR. On the right, we present the histogram showing the velocity distribution of galaxies—solid histogram for all member galaxies, and filled histogram for star-forming galaxies only. (d) Same as in panel (c); galaxies with either spectroscopic or photometric redshifts are plotted.

(A color version of this figure is available in the online journal.)

with high SSFR are located preferentially in the outer region of the cluster (Figures 13(a) and (b)), reflecting the well-known morphology-density relation. There are a few galaxies with high SSFRs near the cluster center, but they have mostly redshifts much lower than the mean redshift of cluster (blue points in the center of Figure 13(a)). The $R_{\mathrm{cl}}-v$ plot of star-forming galaxies (Figures 13(c) and (d)) clearly confirms the trend that galaxies with high SSFR are either located at the outer region of A2255 or at the blue velocity peak.

In order to analyze the distribution of galaxies with high SSFR in more detail, we investigate SSFR as a function of environmental parameters in Figure 14. Figure 14(a) shows $R_{\mathrm{cl}}$ versus SSFR plot. The average SSFR does not change much as a function of $R_{\mathrm{cl}}$, i.e., there is no clear sign of enhancement in SSFR as a function of $R_{\mathrm{cl}}$. Figure 14(b) shows how SSFR changes as a function of local galaxy surface density, $\Sigma_{5 \text { th }}$. Previous works of intermediate redshift clusters suggested that SSFR is enhanced at the intermediate density region of clusters and the enhanced activity is related to the infall of galaxies into the gravitational potential of clusters (e.g., Koyama et al. 2010). For A2255, we find that there is no clear enhancement of SSFR from surface density of $\Sigma_{5 \text { th }}=0.5-2.5 \mathrm{Mpc}^{-2}$. There is a slight increase in the lower density region, at $\Sigma_{5 \text { th }}=0.5 \mathrm{Mpc}^{-2}$, yet the amount of difference is less than a factor of 1.5. Therefore, we conclude that star formation is not enhanced in dense regions of A2255.

We also examine if star formation activities are enhanced at substructures as suggested from studies of other clusters (e.g., Koyama et al. 2010). Figure 14(c) shows the average SSFR for the substructures identified in Section 5.1, in comparison to galaxies in the other areas of the cluster. Errors for the average SSFR are the standard deviations of SSFR are derived through bootstrapping. We find that there is little difference between the average SSFR of galaxies in substructures (Sub) and those not in substructures $(\mathrm{N})$. Several substructures $(\mathrm{A}, \mathrm{W}$, and $\mathrm{Y})$ contain only one or no star-forming galaxy. We conclude that SSFR is not particularly enhanced in substructures even if some galaxies in substructures show high SSFR compared to global average.

Although we find no convincing evidence for the enhancement of SSFR at a particular environment (except that SSFRs are higher at the outer region), we find an interesting trend in the distribution of star-forming galaxies in the inner region. As seen in Figure 13(c), the redshift distribution of star-forming galaxies (filled histogram) is bimodal: a blue velocity peak at $\langle z\rangle=0.077$ and a red velocity peak at $\langle z\rangle=0.082$. (Note that this analysis is only possible for samples with spectroscopic redshifts since the accuracy of photometric redshifts is too low.) At $R_{\mathrm{cl}}<0.5 \mathrm{Mpc}$, the majority of galaxies with high SSFR 

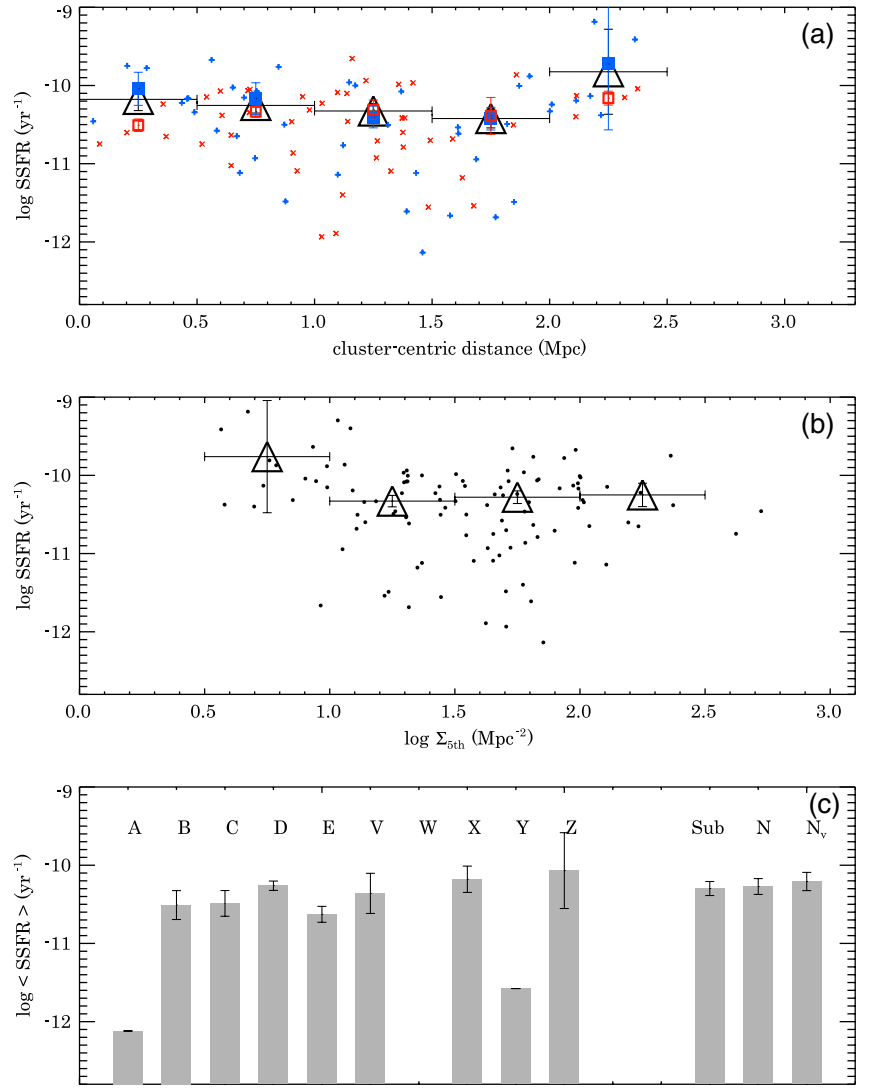

Figure 14. Top: Specific SFR (SSFR) of galaxies as a function of clustercentric radius. The triangles indicate the average SSFR at each bin of clustercentric distance, with error bars produced by bootstrapping. The blue filled squares represent average SSFR from star-forming galaxies at blue velocity peak $(z<0.08)$, and the red open squares represent average SSFR from star-forming galaxies at red velocity peak $(z>0.08)$. Each blue and red cross indicates individual galaxy with either blue or red velocity. Middle: SSFR of galaxies as a function of local surface density of galaxies $\left(\Sigma_{5 \text { th }}\right)$. Bottom: the average SSFR for galaxies brighter than $r=19.0 \mathrm{mag}$ in each substructure (A-Z), in all substructure (Sub), and outside the substructures $(\mathrm{N})$. The error bars are again derived through bootstrapping. Points for substructure $\mathrm{A}$ and $\mathrm{Y}$ are generated by only one galaxy, therefore we do not mark error bars. For galaxies outside the substructures, average SSFR of those with velocities at the blue/red end of the velocity distribution is marked as $N_{v}(z<0.075$ or $z>0.085)$.

(A color version of this figure is available in the online journal.)

belong to the blue velocity peak. We show the average SSFR of galaxies at the blue velocity peak and at the red velocity peak separately in Figure 14(a). Figure 14(a) indicates that the average SSFR of galaxies at the blue velocity peak $(z<0.08)$ is higher than that of galaxies at the red velocity peak $(z>0.08)$ by a factor of $\sim 3$, at the innermost region with $R_{\mathrm{cl}}<0.5 \mathrm{Mpc}$. By randomly selecting and assigning galaxies at $R_{\mathrm{cl}}<0.5 \mathrm{Mpc}$ to either blue or red peaks, we find that the chance probability of finding this observed difference in the average SSFR is less than $5 \%$. This trend appears to continue out to $R_{\mathrm{cl}} \sim 1.0 \mathrm{Mpc}$, but the trend is not statistically significant compared to what we found at $R_{\mathrm{cl}}<0.5 \mathrm{Mpc}$.

\subsection{Environmental Dependence of Galaxy Transformation}

As we discussed in Section 3.4, intermediate MIR-excess galaxies are probes of galaxy transformation from star-forming galaxies (strong MIR excess) to quiescent galaxies (weak MIR excess). By studying the environment of intermediate MIR-excess galaxies, we can gain insight into where in the cluster the galaxy transformation is taking place and what physical mechanisms govern the transformation. The twodimensional spatial distribution of weak/intermediate MIRexcess galaxies is plotted in Figures 15(a) and (b). The plots show that weak MIR-excess galaxies populate near the cluster center, while intermediate MIR-excess galaxies prefer $R_{\mathrm{cl}}>$ $0.5 \mathrm{Mpc}$. We show the trend in a more quantitative way in Figure 16, by presenting the number ratios of galaxies with different levels of MIR-excess as a function of the environmental parameters. Figure 16(a) illustrates the ratio of intermediate MIR-excess galaxies to weak MIR-excess galaxies as a function of the clustercentric radius. Figure 16(b) illustrates the same number ratio as a function of the local galaxy surface density. These are a clearer form of Figure 17, showing the relative fraction of strong/intermediate/weak MIR-excess galaxies as a function of environment, with emphasis on the weak and intermediate MIR-excess galaxies. Figures 17(b) and (d) clearly show that the weak MIR-excess galaxies dominate at the highest densities, and the strong MIR-excess galaxies dominate at the lowest densities. The intermediate MIR-excess galaxies populate at around $\Sigma_{5 \mathrm{th}} \sim 2.0 \mathrm{Mpc}^{-2}\left(R_{\mathrm{cl}} \sim 0.5 \mathrm{Mpc}\right)$, but their number disappears quickly at $\Sigma_{5 \text { th }}<1.5 \mathrm{Mpc}^{-2}$ $\left(R_{\mathrm{cl}} \sim 1.6 \mathrm{Mpc}\right)$. The relative fraction of intermediate MIRexcess to weak MIR-excess galaxies is the highest at the similar density $/ R_{\mathrm{cl}}, \Sigma_{5 \mathrm{th}} \sim 1.5 \mathrm{Mpc}^{-2}$ and $R_{\mathrm{cl}} \sim 1.3 \mathrm{Mpc}$ (Figures 16 (a) and (b)). From this, we conclude that galaxies in transformation (as represented by intermediate MIR-excess galaxies) populate the intermediate density regions (or the clustercentric distance of $R_{\mathrm{cl}}=0.6-1.6 \mathrm{Mpc}$ ). This clustercentric radius corresponds to $0.3-0.8 R_{\text {vir }}$, which exactly coincides with the location in clusters where the ram-pressure stripping is known to be efficient in quenching the star formation in galaxies by stripping their gas reservoir based on the sphere of influence argument (Treu et al. 2003). This is also consistent with the result found in other galaxy clusters (A3112; Braglia et al. 2010).

As in the case of star-forming galaxies, a significant number of intermediate MIR-excess galaxies have redshifts at blue velocity component (Figure 15). The difference in the case of star-forming galaxies is that intermediate MIR-excess galaxies at blue velocity peaks are located at $R_{\mathrm{cl}}>1 \mathrm{Mpc}$. We also examine the correlation between substructures and the fraction of intermediate MIR-excess galaxies (Figure 16(c)). As in the case of star-forming galaxies, there is no clear evidence that intermediate MIR-excess galaxies are preferentially located in the substructures. Note that, however, as we mentioned in Section 3.2, the observation of MIR-excess galaxies is limited by the depth of MIR images-especially at weak/intermediate MIR excess for regions without $S 11$-band coverage. The substructures B, $\mathrm{C}, \mathrm{D}$, and $\mathrm{E}$ are such substructures, and thus the reason we do not find many weak/intermediate MIR-excess galaxies in these substructures may be due to the shallow depth of the $24 \mu \mathrm{m}$ image. Most of the galaxies in substructures B, C, D, and E have $r$-band magnitudes of $16.5<r<18 \mathrm{mag}$, therefore only objects with $z-24 \mu \mathrm{m}>-1$, i.e., close to strong MIR-excess galaxies can be detected as intermediate MIR-excess galaxies.

\section{DISCUSSION}

The MIR data presented so far invoke several interesting points regarding the evolution of galaxies in a cluster environment like A2255, i.e., how cluster-scale dynamics correlates with the evolution of individual member galaxies. In Section 4.2, we show that the total SFR of A2255 is comparable with that of other galaxy clusters of similar mass and dynamical stage, which implies that the cluster-scale merging activity in A2255 

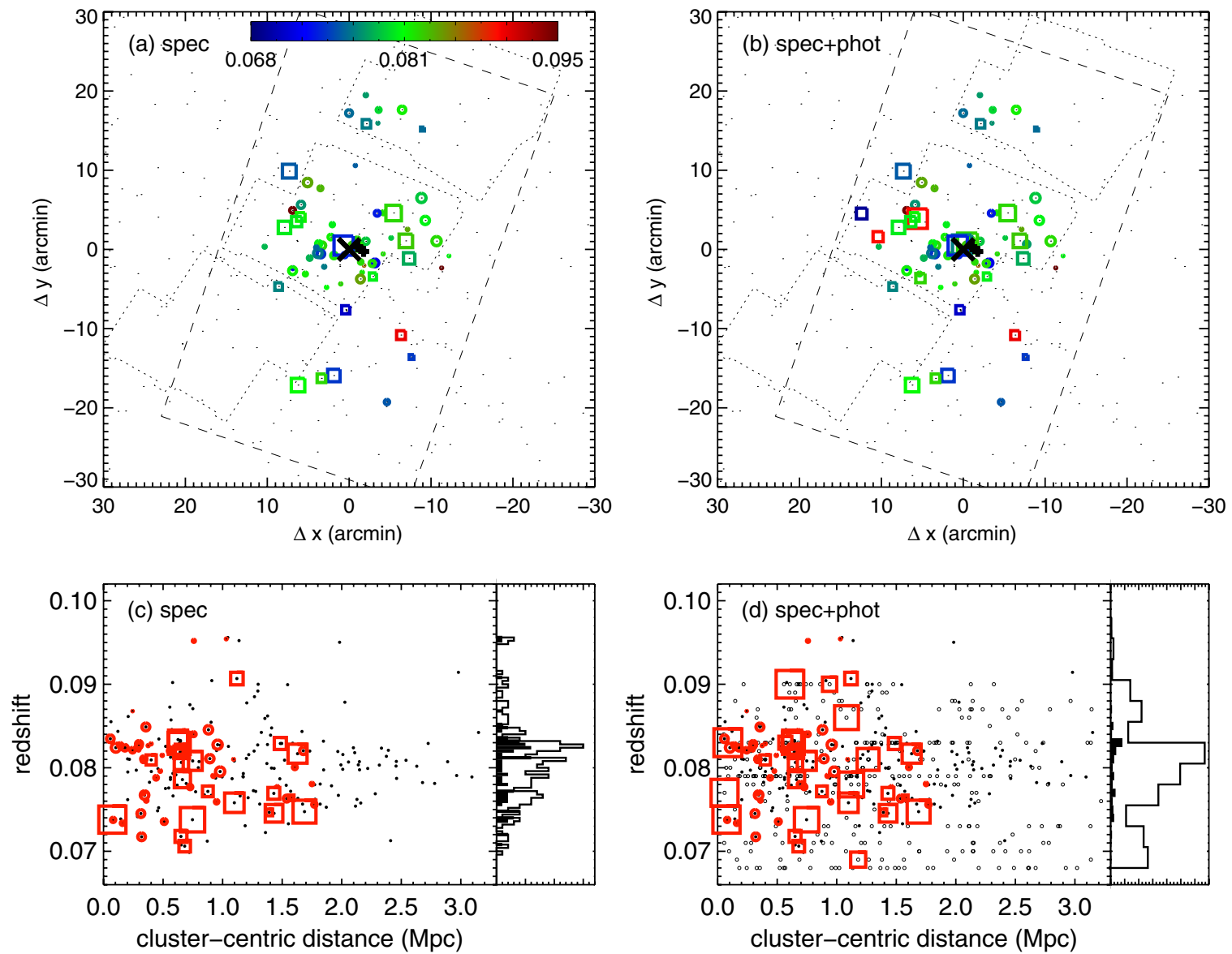

Figure 15. (a) Two-dimensional spatial distribution of weak/intermediate MIR-excess galaxies in A2255. The circles indicate weak MIR-excess galaxies, and the squares indicate intermediate MIR-excess galaxies. The symbol color denotes the redshift of the galaxy as indicated in the color bar, and the symbol size is proportional to the $N 3-S 11$ color. In the case of $\operatorname{mag}(z)-\operatorname{mag}(24 \mu \mathrm{m})$ color, the value is converted to $N 3-S 11$ according to the relation in Figure 3(b). (b) Same as in panel (a); galaxies with either spectroscopic or photometric redshifts are plotted. (c) The clustercentric distance and velocity distribution of weak/intermediate galaxies in A2255. The points plotted as small dots are all member galaxies with spectroscopic redshifts defined in the Y03 catalog. Overplotted are weak(circle)/intermediate(square) MIR-excess galaxies, with symbol size proportional to the $N 3-S 11$ color. On the right, we present the histogram showing the velocity distribution of galaxies-solid histogram for all member galaxies, and filled histogram for weak/intermediate MIR-excess galaxies only. (d) Same as in panel (c); galaxies with either spectroscopic or photometric redshifts are plotted.

(A color version of this figure is available in the online journal.)

does not enhance the star formation activity of member galaxies to the level of galaxies in some of the active clusters at intermediate redshifts. There is no clear evidence that substructures are closely associated with galaxies with high SSFR (Section 5.2, Figure 14(c)). Star-forming galaxies are located at the outer part of the cluster $\left(R_{\mathrm{cl}}>0.5 \mathrm{Mpc}\right)$ or blue/red end of the velocity distribution (Figure 13). While the dynamical status of A2255 is considered to be "post-merger" from the previous X-ray observations - no distinct multiple temperature peak, the temperature gradient elongated in east-west direction (Davis et al. 2003)_our results are consistent with no enhancement of star formation related to the cluster merger.

This result is at odds with conclusions put forward in previous studies. Miller \& Owen (2003) suggested that the fraction of radio selected star-forming galaxies is twice larger in A2255 compared to other galaxy clusters including Coma cluster. They ascribed this "enhanced" star formation in A2255 to the cluster-cluster merger, arguing that the alignment of starforming galaxies in the north-south direction is a product of cluster-cluster merging in the east-west direction. However, as discussed earlier, we neither find such an alignment of starforming galaxies nor more active star formation than in the Coma cluster. The discrepancy between their results and ours can be understood as follows. Our MIR observation discovers more star-forming galaxies in A2255 than Miller \& Owen (2003), since we are probing the SFR much deeper than their SFR detection limit of $\sim 2.5 M_{\odot} \mathrm{yr}^{-1}$. All the radio-selected star-forming galaxies in Miller \& Owen (2003) are detected in MIR if they are within either the AKARI or the Spitzer fields. On the other hand, a number of strong MIR-excess galaxies having comparable SFR with radio-selected star-forming galaxies are not detected in radio. Therefore, we conclude that the difference between the MIR study and the radio study arises from the way star-forming galaxies are selected, and that the effect of the environment on star formation activities requires a deep, multiwavelength data so as not to miss galaxies with moderate SFR. In another work, Yuan et al. (2005) suggested that cluster-cluster merging in A2255 had different effects on star-forming galaxies with different morphologies, i.e., SFRs of late-type galaxies increase toward the center and the SFRs of the early-type galaxies decrease toward the center using the SFR measured in optical spectroscopy, concluding that the cluster-cluster merging activity triggered star formation in the central region of the cluster. However, our study shows no marked increase in the SSFRs of galaxies near the cluster center. Furthermore, star-forming galaxies near the cluster center are found to have 

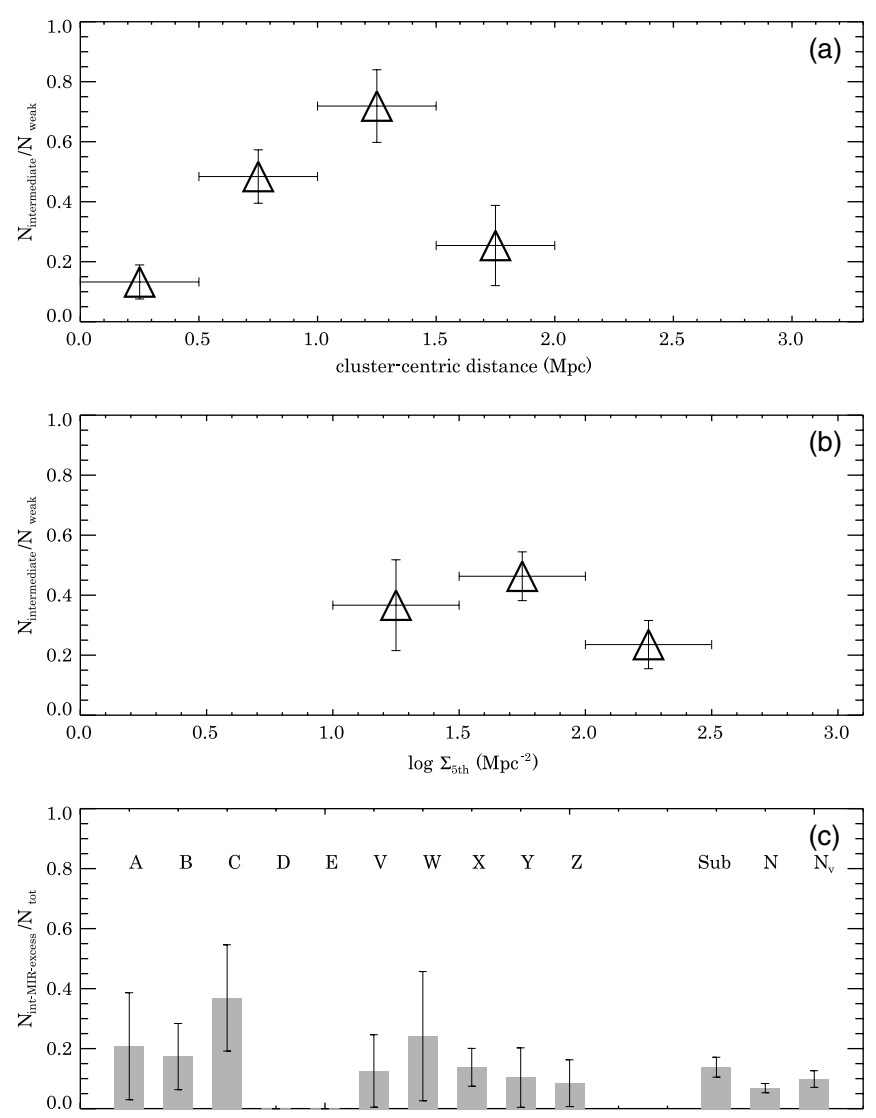

Figure 16. Top: the ratio between the number of intermediate MIR-excess galaxies and the number of weak MIR-excess galaxies, as a function of clustercentric radius. The values are derived using $r<19.0 \mathrm{mag}$ galaxies. The error bars are derived through bootstrapping. Middle: the ratio between the number of intermediate MIR-excess galaxies and the number of weak MIRexcess galaxies, as a function of local surface density of galaxies. Again, the values are derived using $r<19.0$ mag galaxies, and the error bars indicate bootstrapping errors. Bottom: the fraction of intermediate MIR-excess galaxies among the "total" galaxies in substructures, and outside the substructures $\left(N_{\text {tot }}\right.$ indicates the total number of galaxies brighter than $r=19.0$ mag in each substructure, or outside the substructures, including all levels of MIR-excess galaxies and MIR non-excess galaxies). The marks for substructures $\mathrm{A}-\mathrm{Z}$ and galaxies outside the substructures $(\mathrm{N})$, galaxies outside the substructures with extreme velocities $\left(\mathrm{N}_{v}\right)$ are the same as in Figure 14.

significantly blueshifted radial velocities with respect to the mean cluster redshift whose origin is discussed in more detail below.

We can gain another valuable insight into the galaxy evolution in a merging cluster from the velocity distribution of MIRexcess galaxies. In Sections 5.2 and 5.3, we noted a high fraction of star-forming galaxies and intermediate MIR-excess galaxies in the blue velocity peak and the lack of star-forming galaxies with high SSFR at the redshift peak especially at $R_{\mathrm{cl}}<0.5 \mathrm{Mpc}$. We discuss here how this interesting tendency can arise. A simulation performed by Fujita et al. (1999) of a merging cluster illustrates how galaxies in the merging cluster evolve as the merging of the clusters proceed. According to their work, the merging activity increases the ram pressure, causing more efficient stripping of gas from galaxies in the cluster, hence quenching star formation rather than enhancing it. But at the same time, their simulation indicates that some cluster galaxies associated with the merging can survive the ram-pressure stripping and maintain their blue colors if their relative velocities to the cluster center are high, simply because they pass through the cluster too fast for the ram pressure to quench the star formation. Due to this effect, the relative velocities of blue galaxies are larger than those of red galaxies by about $\sim 1000-1500 \mathrm{~km} \mathrm{~s}^{-1}$ in a merging cluster at $3.6 \mathrm{Gyr}$ after the core-crossing. The outcome of the simulation matches well with our results-i.e., no particular enhancement of star formation activities and the peculiar distribution of strong/ intermediate MIR-excess galaxies that are found preferentially at the blue velocity peak. Therefore, we suggest that the discovery of the preferential occupation of the blue velocity peak by strong/intermediate MIR-excess galaxies is supporting evidence of galaxy transformation taking place in a merging cluster following the scenario presented in Fujita et al. (1999).

These key findings allow us to draw a global picture of the galaxy evolution process taking place in A2255. As witnessed from the strong/intermediate MIR-excess galaxies at the blue velocity peak and the lack of notable enhancement of star formation both globally in the cluster and in substructures, the cluster-cluster merging suppresses the star formation activity with the ram-pressure stripping rather than enhancing it. The quenching of star formation happens two-fold, one related to the cluster-cluster merging, and another related to the rampressure stripping of gas in (infalling) galaxies at $0.3 R_{\mathrm{vir}}<$ $R_{\mathrm{cl}}<R_{\text {vir }}$ where the supporting evidence comes from the spatial distribution of intermediate MIR-excess galaxies and the lack of star-forming galaxies at $R_{\mathrm{cl}}<0.5 \mathrm{Mpc}$.

\section{CONCLUSION}

We investigated the MIR properties of galaxies in the merging cluster A2255 using the AKARI MIR observation over 3-24 $\mu \mathrm{m}$ in addition to the Spitzer MIPS 24, $70 \mu \mathrm{m}$ data in order to understand the ongoing SFR activities in such a cluster, and the role of the environment on the subsequent evolution of the star-forming galaxies into red, quiescent galaxies.

As a way to trace the evolutionary sequence of galaxies from the star-forming stage through the transition stage to the dead, quiescent stage, we examined the MIR colors $(N 3-S 11$ or $z-24 \mu \mathrm{m})$ of the cluster member galaxies. We found that MIR colors of the cluster galaxies show a large dispersion unlike the tight red or blue sequences in the optical or NIR color-magnitude relation. Virtually almost all $(>90 \%)$ of the member galaxies with MIR detection have redder $N 3-S 11$ colors than expected from stellar photospheric emission only. The MIR excess can be categorized into three classes according to $N 3-S 11$ (or $z-24 \mu \mathrm{m}$ ) color; the first is a population of galaxies forming a blue sequence at $\langle N 3-S 11\rangle \sim-1.7$ ("weak" MIR-excess) that can be considered as a coeval population with the dust emission coming from the circumstellar dust around AGB stars. The second case is a population with a "strong" MIR excess $(N 3-S 11>0.2)$, most of which are starforming galaxies with blue optical colors. The third case is an "intermediate" MIR-excess population $(-1.2<N 3-S 11<$ 0.2 ) which lies between the weak and the strong MIR-excess galaxies. These are mostly passive galaxies with a young mean stellar age. As such, the MIR color works as a classifier of galaxies at different stages of evolution. The intermediate MIRexcess galaxies have late-type morphology ( $\sim 80 \%)$, while the weak MIR-excess galaxies are predominantly early-type $(\sim 80 \%)$. The strong MIR-excess galaxies are mostly late-type galaxies. The morphologies of the different MIR-excess classes support the idea that the intermediate MIR-excess galaxies are the transition population bridging the strong and the weak MIRexcess galaxies. 

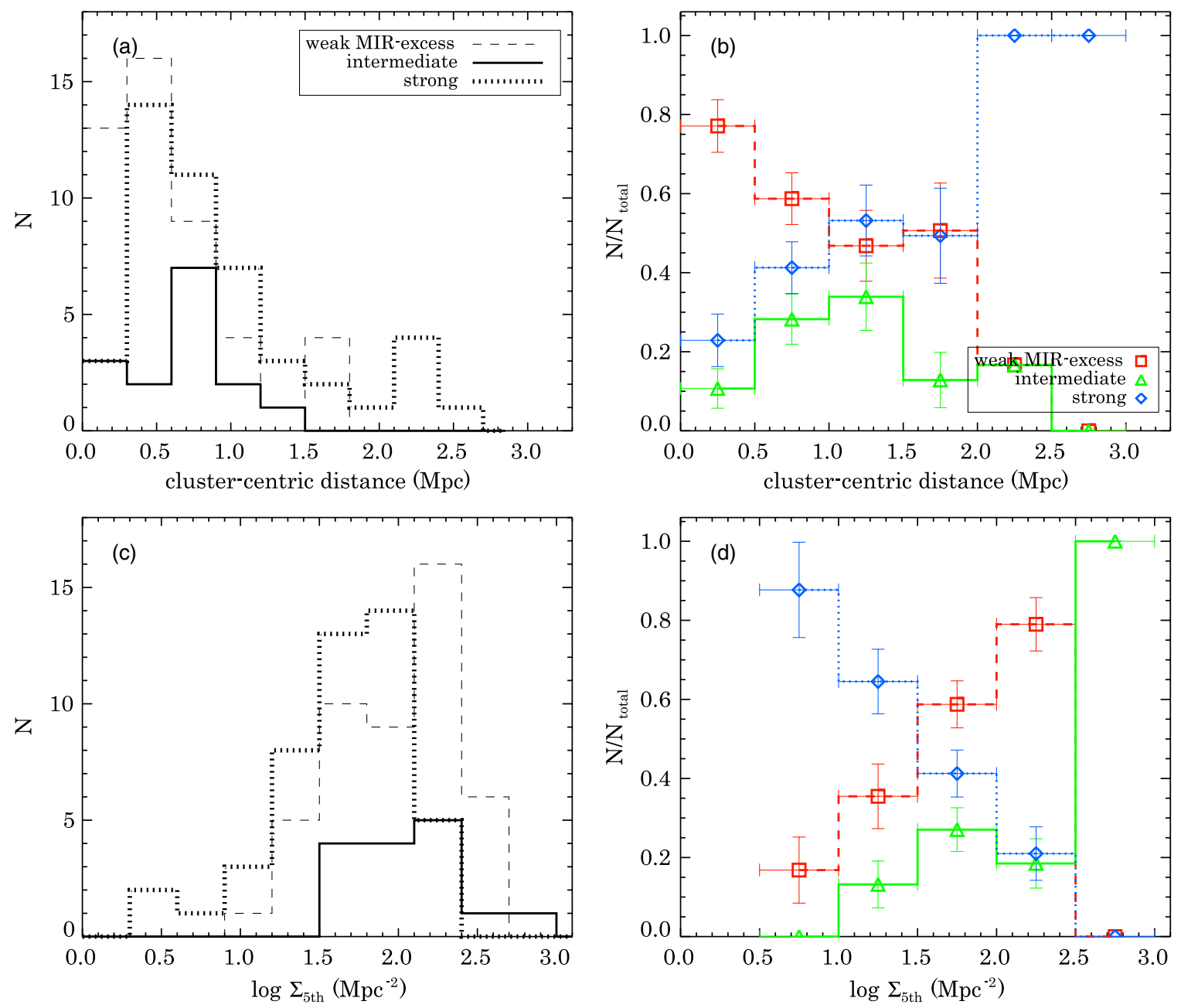

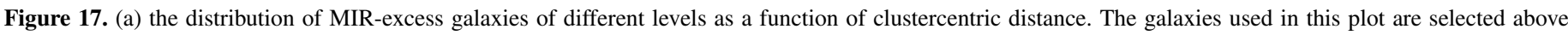

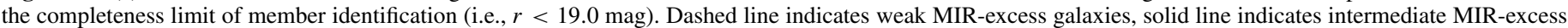

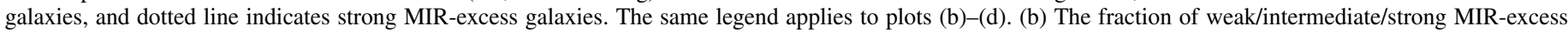

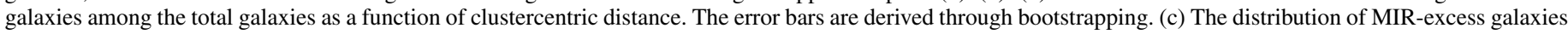

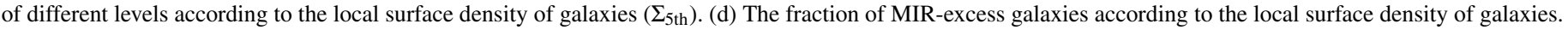

(A color version of this figure is available in the online journal.)

Armed with the three different classes of MIR-excess galaxies which trace different stages of the galaxy evolution, we addressed whether the star formation is enhanced in the merging cluster or not, and how the environment affects the galaxy transition. Using star-forming galaxies as represented by strong MIR-excess galaxies, we derived the total SFR of A2255. The IR luminosities of individual galaxies has the range $6.0 \times 10^{8} L_{\odot}<$ $L_{\mathrm{IR}}<3.2 \times 10^{10} L_{\odot}$, with the total SFR for the entire cluster being $\sim 130 M_{\odot} \mathrm{yr}^{-1}$. The integrated SFR, and the IR luminosity function of A2255, is consistent with those of other galaxy clusters at similar redshifts and with similar masses. This supports the idea that cluster scale dynamics (cluster-cluster merging) does not enhance the star formation activity. Yet, it should be noted that most available galaxy clusters studied to date are biased towards dynamically unrelaxed systems such as A2255.

We identify substructures and two distinct velocity components (the blue velocity peak and the red velocity peak) in A2255. To understand how large-scale (cluster-cluster) and small-scale (group/galaxy infall) merging activities affect the galaxy evolution, the star formation and its quenching of MIRexcess galaxies were examined in these distinct components in A2255 as well as a function of the clustercentric distance and the local density. Star-forming galaxies are not only preferentially located in the outer, lower density part of the cluster, but are also found to be more abundant in the blue velocity peak. The latter fact has been predicted in simulations of merging clusters, where galaxies in the highest velocity component suffer less quenching from ram-pressure stripping. No marked increase in the specific star formation is found over the cluster region. The relative fraction of intermediate MIR-excess galaxies increases at the intermediate density region or at regions with clustercentric distances of $0.5-2.0 \mathrm{Mpc}$, suggesting that quenching in star formation occurs over such a region where rampressure stripping has been speculated to be a main star formation suppression mechanism. These findings support the idea that the cluster-scale merging suppresses the star formation and that ram-pressure stripping is a main mechanism of star formation quenching in A2255.

We thank L. Piovan for providing his SED model. We thank R. Gobat and J. H. Lee for helpful discussions. This work is based on observations with $A K A R I$, a JAXA project with the participation of ESA. The AKARI images are obtained from a Mission Program CLEVL (CLusters of galaxies EVoLution 
studies). This work was supported by the Korea Science and Engineering Foundation (KOSEF) grant No. 2009-0063616, funded by the Korea government (MEST).

\section{REFERENCES}

Abadi, M. G., Moore, B., \& Bower, R. G. 1999, MNRAS, 308, 947

Abazajian, K., et al. 2004, AJ, 128, 502

Abazajian, K., et al. 2009, ApJS, 182, 543

Abell, G. O. 1958, ApJS, 3, 211

Bai, L., Rieke, G. H., \& Rieke, M. J. 2007, ApJ, 664, 181

Bai, L., Rieke, G. H., Rieke, M. J., Christlein, D., \& Zabludoff, A. I. 2009, ApJ, 693, 1840

Balogh, M. L., Morris, S. L., Yee, H. K. C., Carlberg, R. G., \& Ellingson, E. 1999, ApJ, 527, 54

Beers, T. C., Geller, M. J., \& Huchra, J. P. 1982, ApJ, 257, 2

Bekki, K. 1999, ApJ, 510, L15

Bell, E. F. 2003, ApJ, 586, 794

Bertin, E., \& Arnouts, S. 1996, A\&AS, 117, 393

Boselli, A., \& Gavazzi, G. 2006, PASP, 118, 517

Braglia, F. G., et al. 2010, (arXiv:1003.2629)

Brand, K., et al. 2009, ApJ, 693, 340

Bressan, A., et al. 2007, in ASP Conf. Ser. 374, From Stars to Galaxies: Building the Pieces to Build Up the Universe, ed. A. Vallenari et al. (San Francisco, CA: ASP), 333

Brinchmann, J., Charlot, S., White, S. D. M., Tremonti, C., Kauffmann, G., Heckman, T., \& Brinkmann, J. 2004, MNRAS, 351, 1151

Burns, J. O., Roettiger, K., Pinkney, J., Perley, R. A., Owen, F. N., \& Voges, W. 1995, ApJ, 446, 583

Calzetti, D., Armus, L., Bohlin, R. C., Kinney, A. L., Koornneef, J., \& StorchiBergmann, T. 2000, ApJ, 533, 682

Chary, R., \& Elbaz, D. 2001, ApJ, 556, 562

Choi, P. I., et al. 2006, ApJ, 637, 227

Davis, D. S., Miller, N. A., \& Mushotzky, R. F. 2003, ApJ, 597, 202

Davis, D. S., \& White, R. E., III. 1998, ApJ, 492, 57

Donnelly, R. H., Forman, W., Jones, C., Quintana, H., Ramirez, A, Churazov, E., \& Gilfanov, M. 2001, ApJ, 562, 254

Dressler, A., \& Gunn, J. E. 1983, ApJ, 270, 7

Elíasdóttir, A., \& Möller, O. 2007, J. Cosmol. Astropart. Phys., JCAP07(2007)006

Feretti, L., Boehringer, H., Giovannini, G., \& Neumann, D. 1997, A\&A, 317, 432

Fujita, Y. 1998, ApJ, 509, 587

Fujita, Y., Takizawa, M., Nagashima, M., \& Enoki, M. 1999, PASJ, 51, 1

Fukugita, M., Shimasaku, K., \& Ichikawa, T. 1995, PASP, 107, 945

Gallazzi, A., Charlot, S., Brinchmann, J., \& White, S. D. M. 2006, MNRAS, 370,1106

Gallazzi, A., Charlot, S., Brinchmann, J., White, S. D. M., \& Tremonti, C. A. 2005, MNRAS, 362, 41

Gladders, M. D., Lopez-Cruz, O., Yee, H. K. C., \& Kodama, T. 1998, ApJ, 501, 571

Goto, T. 2007, MNRAS, 381, 187

Gunn, J. E., \& Gott, J. R., III. 1972, ApJ, 176, 1
Hwang, H. S., \& Lee, M. G. 2009, MNRAS, 397, 2111

Im, M., Griffiths, R. E., \& Ratnatunga, K. U. 1997, ApJ, 475, 457

Im, M., et al. 2008, in ASP Conf. Ser. 399, Panoramic Views of Galaxy Formation and Evolution, ed. T. Kodama, T. Yamada, \& K. Aoki (San Francisco, CA: ASP), 382

Kauffmann, G., et al. 2003, MNRAS, 341, 33

Kennicutt, R. C. 1998, ARA\&A, 36, 189

Ko, J., et al. 2009, ApJ, 695, L198

Koyama, Y., Kodama, T., Shimasaku, K., Hayashi, M., Okamura, S., Tanaka, I., \& Tokoku, C. 2010, MNRAS, 403, 1611

Lacy, M., et al. 2004, ApJS, 154, 166

Lavery, R. J., \& Henry, J. P. 1994, ApJ, 426, 524

Lee, M. G., et al. 2009, in ASP Conf. Ser. 418, AKARI, A Light to Illuminate the Misty Universe, ed. Takashi Onaka et al. (San Francisco, CA: ASP), 241

Lokas, E. L., \& Mamon, G. A. 2003, MNRAS, 343, 401

Lorente, R., Onaka, T., Ita, Y., Ohyama, Y., \& Pearson, C. P. 2007, AKARI IRC Data User Manual Version 1.2

Marcillac, D., Rigby, J. R., Rieke, G. H., \& Kelly, D. 2007, ApJ, 654, 825

Markevitch, M., Forman, W. R., Sarazin, C. L., \& Vikhlinin, A. 1998, ApJ, 503, 77

Meurer, G. R., Heckman, T. M., \& Calzetti, D. 1999, ApJ, 521, 64

Miller, N. A., \& Owen, F. N. 2003, AJ, 125, 2427

Moore, B., Katz, N., Lake, G., Dressler, A., \& Oemler, A. 1996, Nature, 379, 613

Mullis, C. R., Henry, J. P., Gioia, I. M., Böhringer, H., Briel, U. G., Voges, W., \& Huchra, J. P. 2001, ApJ, 553, L115

Murakami, H., et al. 2007, PASJ, 59, 369

Navarro, J. F., Frenck, C. S., \& White, S. D. M. 1997, ApJ, 490, 493

Neumann, D. M. 2005, A\&A, 439, 465

Onaka, T., et al. 2007, PASJ, 59, 401

Osterbrock, D. E. 1989, Astrophysics of Gaseous Nebulae and Active Galactic Nuclei (Mill Valley, CA: University Science Books)

Park, C., \& Hwang, H. S. 2009, ApJ, 699, 1595

Piovan, L., Tantalo, R., \& Chiosi, C. 2003, A\&A, 408, 559

Poggianti, B. M., Smail, I., Dressler, A., Couch, W. J., Barger, A. J., Butcher, H., Ellis, R. S., \& Oemler, A., Jr. 1999, ApJ, 518, 576

Poggianti, B. M., et al. 2009, ApJ, 697, L137

Quillen, A. C., Rieke, G. H., Rieke, M. J., Caldwell, N., \& Engelbracht, C. W. 1999, ApJ, 518, 632

Sauvageot, J. L., Belsole, E., \& Pratt, G. W. 2005, A\&A, 444, 673

Shim, H., Im, M., Choi, P., Yan, L., \& Storrie-Lombardi, L. 2007, ApJ, 669, 749

Stern, D., et al. 2005, ApJ, 631, 163

Strauss, M. A., et al. 2002, AJ, 124, 1810

Struble, M. F., \& Rood, H. J. 1999, ApJS, 125, 35

Tran, K. H., van Dokkum, P., Illingworth, G. D., Kelson, D., Gonzalez, A., \& Franx, M. 2005, ApJ, 619, 134

Treu, T., Ellis, R. S., Kneib, J.-P., Dressler, A., Smail, I., Czoske, O., Oemler, A., \& Natarajan, P. 2003, ApJ, 591, 53

Watanabe, M., Yamashita, K., Furuzawa, A., Kunieda, H., Tawara, Y., \& Honda, H. 1999, ApJ, 527, 80

Yuan, Q., Zhao, L., Yang, Y., Wen, Z., \& Zhou, X. 2005, AJ, 130, 2559

Yuan, Q., Zhou, X., \& Jiang, Z. 2003, ApJS, 149, 53

Zar, J. H. 1972, J. Am. Stat. Assoc., 67, 578 